Chapman University

Chapman University Digital Commons

Philosophy Faculty Articles and Research

Philosophy

$12-5-2018$

Frege's Constraint and the Nature of Frege's Foundational Program

Marco Panza

Andrea Sereni

Follow this and additional works at: https://digitalcommons.chapman.edu/philosophy_articles

Part of the Logic and Foundations of Mathematics Commons, Other Philosophy Commons, and the Philosophy of Science Commons 


\section{Frege's Constraint and the Nature of Frege's Foundational Program}

Comments

This article was originally published in Review of Symbolic Logic, volume 12, issue 1, in 2019. DOI: 10.1017 /

S1755020318000278

\section{Copyright}

Cambridge University Press 


\title{
FREGE'S CONSTRAINT AND THE NATURE OF FREGE'S FOUNDATIONAL PROGRAM
}

\author{
MARCO PANZA \\ CNRS, IHPST, Paris (CNRS and University of Paris 1, Panthéon-Sorbonne), \\ Chapman University \\ and \\ ANDREA SERENI \\ Scuola Universitaria Superiore IUSS Pavia
}

\begin{abstract}
Recent discussions on Fregean and neo-Fregean foundations for arithmetic and real analysis pay much attention to what is called either 'Application Constraint' (AC) or 'Frege Constraint' (FC), the requirement that a mathematical theory be so outlined that it immediately allows explaining for its applicability. We distinguish between two constraints, which we, respectively, denote by the latter of these two names, by showing how AC generalizes Frege's views while FC comes closer to his original conceptions. Different authors diverge on the interpretation of FC and on whether it applies to definitions of both natural and real numbers. Our aim is to trace the origins of FC and to explore how different understandings of it can be faithful to Frege's views about such definitions and to his foundational program. After rehearsing the essential elements of the relevant debate $(\S 1)$, we appropriately distinguish $A C$ from $F C(\S 2)$. We discuss six rationales which may motivate the adoption of different instances of $A C$ and $F C(\$ 3)$. We turn to the possible interpretations of FC ( $\$ 4)$, and advance a Semantic FC (\$4.1), arguing that while it suits Frege's definition of natural numbers (4.1.1), it cannot reasonably be imposed on definitions of real numbers (\$4.1.2), for reasons only partly similar to those offered by Crispin Wright (\$4.1.3). We then rehearse a recent exchange between Bob Hale and Vadim Batitzky to shed light on Frege's conception of real numbers and magnitudes (\$4.2). We argue that an Architectonic version of FC is indeed faithful to Frege's definition of real numbers, and compatible with his views on natural ones. Finally, we consider how attributing different instances of FC to Frege and appreciating the role of the Architectonic FC can provide a more perspicuous understanding of his foundational program, by questioning common pictures of his logicism (\$5).
\end{abstract}

In recent debates on Fregean and neo-Fregean epistemologies of mathematics, much interest has been devoted to what is usually called 'Application Constraint'. Very generally speaking, this is the requirement that a mathematical theory be outlined in a way which immediately allows accounting for its applicability. Arguably, Frege took this requirement as an essential guide for his foundational endeavor, and this has made many Frege scholars follow Wright (2000) in calling it 'Frege's Constraint'.

A large part of the recent discussion has focused on whether this requirement, in one or another of its possible understandings, is able to adjudicate between rival conceptions of the natural and/or real numbers. Our present purpose is not so much to add directly to this

Received: July 27, 2017.

2010 Mathematics Subject Classification: 00A30, 03A05, 03A10.

Key words and phrases: Frege's Constraint, applicability of mathematics, Frege's foundational program, logicism. 
issue. Rather, we want to come back to the origins of the requirement and to explore how different understandings of it can be, respectively, faithful to Frege's views about natural and real numbers, and/or to his logicist project.

In trying to clarify the matter, we suggest to distinguish a quite general requirementwhich will label 'Application Constraint' ('AC')—from a more specific one, which Frege actually endorsed — which we consequently label 'Frege's Constraint' ('FC'). After considering how the latter can be retrieved from Frege's views, we will survey different rationales that may motivate the former, discussing which among them could also drive the latter. This will help us to better hem in FC, to offer a compact formulation of it that will nonetheless allow for different possible specifications or instances, which may be, respectively, motivated by different rationales, and stand out as appropriate as regards different branches of mathematics.

Accordingly, we will submit that two different instances of $\mathrm{FC}-\mathrm{a}$ semantic and an architectonic one, as we will label them ${ }^{1}$ — partly motivated by different rationales, can be retrieved from Frege's approaches to natural and real numbers, respectively. This will bring us to argue that a careful consideration of the ways in which FC commands such approaches leads to a more nuanced understanding of Frege's foundational program than many other interpretations suggest.

We will proceed as follows. In $\S 1$ we will rehearse how FC can be retrieved in Frege's writings; in $\S 2$, we will motivate and substantiate the distinction between $A C$ and FC, by clarifying why the latter can be seen as a specification of the former; in $\S 3$ we will discuss some possible rationales for $\mathrm{AC}$; in $\S 4$, we will focus on the semantic and architectonic instances of FC and on their relations with Frege's treatment of natural and real numbers; finally, in $\$ 5$, we will reconsider Frege's foundational program in the light of these two instances of FC.

\$1. The origins of Frege's Constraint. While Frege's worry for requiring a satisfactory account for applications in a principled way had already been made explicit in Frege (1884), especially in connection with empiricist approaches, it is in Frege's treatment of reals in Frege (1903) that FC is most often detected. ${ }^{2}$ Here is how Hale summarizes the philosophical import of Frege's arguments (Hale, 2000, p. 104):

From a philosophical standpoint, the most striking and most important features of Frege's treatment of the reals are two: (i) the real numbers are to be defined as ratios of quantities ${ }^{3}(\S 73, \S 157)$ and (ii) in regard to the

1 The second of these labels is borrowed from Gandon (2012), §6.3.

2 After having critically discussed at length a number of available definitions of the reals, including Cantor's, Dedekind's, Heine's, Stolz's, Thomae's and Weierstrass's (Frege (1903), $\S$ II.55-\$II.155), Frege informally prospects an alternative definition, which should have identified reals with "ratios of magnitudes [Grössenverhältnis]" (ibidem, §II.156-§II.164). Insofar as he takes magnitudes to belong to different domains, he first proceeds by formally defining "domains of magnitudes [Grössengebiete]" (ibidem, §II.165-§II.245). An additional part of his treatise should have followed, where Frege should have proved the existence of such domains, and defined real numbers as ratios on them, but he did never write it: volume II of Grundgesetze stops with the definition of domains of magnitudes, followed by the well-known Nachwort suggesting a(n illusory) way out from the contradiction, and no third volume ever followed.

3 Hale uses 'quantity' to denote what Frege calls 'Grösse'. We prefer 'magnitude' (in line with the English translators of the Grundgesetze: cf. Frege (2013)). This discrepancy also reappears in cognate terminology. 
analysis of the notion of quantity, the fundamental question requiring to be answered is not: What properties must an object have, if it is to be a quantity? but: What properties must a concept have, if the objects falling under it are to constitute quantities of a single kind? (\$160-\$161).

According to Hale, the first of these two features is, in turn, motivated by FC (ibidem):

[...][Frege's] insistence that reals be defined as ratios of quantities derives from his belief that the application of reals as measures of quantities is essential to their very nature, and so should be built into an adequate definition of them. It is this, more perhaps than any other single consideration, which underlies his dissatisfaction with the theories of Cantor and Dedekind, on which the applicability of the reals appears, in Frege's view, merely as an incidental extra.

A few passages from Frege's discussion of the matter are usually quoted to support such a reading. One is this, taken from his assessment of Cantor's definition (Frege, 2013, §II.75; Frege, 2013, pp. $\left.86_{2}-87_{2}\right):^{4}$

Cantor takes it to be crucial [...] that by means of these abstract magnitudes $b, b^{\prime}, b^{\prime \prime} \ldots$ one can effect a precise quantitative determination of concrete magnitudes proper, for example, a geometric line segment. Thus, far from being merely a pleasing bonus, the application within geometry is crucial. However, if it is crucial, then this goes against Cantor's theory, since what is crucial does not occur in the definition of numerical magnitude. It is only after $b, b^{\prime}, b^{\prime \prime} \ldots$ are introduced that the determination of distances by means of numerical magnitudes is provided. The earlier introduction of numerical magnitudes is purely arithmetical, but does not contain what is crucial; the latter statement, how distances are determined by numerical magnitudes, contains what is crucial, but it is not purely arithmetical. And thus the goal that Cantor set himself is surely missed.

Here is another, taken from Frege's informal presentation of his own definition (Frege, 1903, §II.159; Frege, 2013, pp. 1562-1572):

So the path to be taken here steers between the old approach, still preferred by H. Hankel, ${ }^{5}$ of a geometrical foundation for the theory of the irrational numbers and the approaches pursued in recent times. From the former we retain the conception of a real number as a magnitude-ratio, or measuring number, but separate it from geometry and indeed from all specific kinds of magnitudes, thereby coming closer to the more recent efforts. At the same time, however, we avoid the emerging problems of the latter approaches, that either measurement does not feature at all, or that it features without any internal connection grounded in the nature of the number itself, but is merely tacked on externally, from which it follows that we would, strictly speaking, have to state specifically for

4 Henceforth, we will adopt the standard contemporary translations of Frege's works even if we take the liberty to make minor changes when we think this may be needed in order to be more faithful to the original.

5 Frege's reference is to Hankel (1687). 
each kind of magnitude how it should be measured, and how a number is thereby obtained. Any general criteria for where the numbers can be used as measuring numbers and what shape their application will then take, are here entirely lacking.

So we can hope, on the one hand, not to let slip away from us the ways in which arithmetic is applied in specific areas of knowledge, without, on the other hand, contaminating arithmetic with the objects, concepts, relations borrowed from these sciences and endangering its special nature and autonomy. One may surely expect arithmetic to present the ways in which arithmetic is applied, even though the application itself is not its subject matter.

The attempt will have to show whether our plan is executable. Here the following concern may arise. If the positive square root of 2 is a magnitude-ratio, then in order to define it, it seems necessary to supply magnitudes which have this ratio to each other. But where should we obtain them from, if appeal to geometrical or physical magnitudes is forbidden? And yet we do indeed require such a magnitude-ratio, since otherwise even the sign ' $\sqrt{2}$ ' may not be used.

The crucial role of FC in Frege's treatment both of natural and reals has been emphasized by Dummett. The following remark will be relevant in our discussion (Dummett, 1991, pp. 272-273):

A correct definition of the natural numbers must, on his [namely, Frege's] view, show how such a number can be used to say how many matches there are in a box or books on a shelf. Yet number theory has nothing to do with matches or with books: its business in this regard is only to display what, in general, is involved in stating the cardinality of the objects, of whatever sort, that fall under some concept, and how the natural numbers can be used for their purpose. In the same way, analysis has nothing to do with electric charge or mechanical work, with length or temporal duration; but it must display the general principle underlying the use of the real numbers to characterize the magnitude of quantities of these and other kinds. A real number does not directly represent the magnitude of a quantity, but only the ratio of one quantity to another of the same type; and this is in common to all the various types [...]. It is what is in common to all such uses, and only that, which must be incorporated into the characterization of the real numbers as mathematical objects: that is how statements about them can be allotted a sense which explains their applications, without violating the generality of arithmetic by allusion to any specific type of empirical application. ${ }^{6}$

6 Although Dummett's remarks certainly emphasize the basic theoretical point Frege makes in the passages quoted above, they are silent on another relevant aspect of the latter, namely on how they locate Frege within the 19th-century discussion about the foundation of analysis. Just two pages after the latter of these texts (Frege, 1903, §II.162), Frege extensively quotes a passage from Gauss's 1831 announcement of his Theoria residuorum biquadraticorum - Commentatio secunda (Gauss (1831, p. 635)). This is part of a larger discussion about "general arithmetic [allgemeine Arithmetik]" and the legitimacy of negative and imaginary numbers. Concerning the former, Gauss argues that "Positive and negative numbers can find application only [...] when 
The two passages quoted above, as well as others where Frege makes similar points, ${ }^{7}$ and the very structure of Frege's envisaged definition of real numbers, ${ }^{8}$ unquestionably justify both Dummett's and Hale's claims about the centrality of FC regarding such definition.

what is counted are not substances (conceivable objects [denkbare Gegenstände]) but instead relations between each two objects", so that the positive vs. negative opposition does merely concern "the members of the relation in such a way that if the relation [...] from $A$ to $B$ counts as +1 , then the relation from $B$ to $A$ must be represented as -1 " (Frege, 1903, §II.162; Frege, 2013, pp. $159_{2}$ ). The reference to counting makes clear that Gauss is here thinking of integer numbers. Frege expands his views to positive and negative magnitudes (considering it to be useless to take absolute ones into account: see footnote 42, below), and builds on this remark to suggest that these are to be considered as extensions of relations. This specific suggestion apart, his quoting this passage emphasizes his attention to the question Gauss is discussing there, namely how to ascribe some sort of (objective) "reality" (the terms is Gauss') to items to be studied within "general arithmetic". The previous passages make Frege's attitude towards this question clear. Surely, the proposal to separate the "conception of a real number as a magnitude-ratio, or measuring number [Auffassung der reellen Zahl als Grössenverhältnis oder Maasszahl]" from "all specific kinds of magnitudes [allen besondern Grössenarten]" and to look for a general notion of a magnitude (or, better, as we will see later, of a domain of magnitudes) is unquestionably reminiscent of Riemann's idea that different "notions of magnitudes [Grössenbegriffe] are only possible where there is an antecedent general notion [ein allgemeiner Begriff] which admits of different specializations [Bestimmungsweisen]", each of which "is capable of different measuring-relations [Massverhältnisse]” (Riemann, 1866-1867, pp. 133-135; Riemann, 2016, pp. 31-32). It remains, however, that Frege's perspective is quite different from Riemann's. Where Riemann delineates an informal characterization of a "manifold [Mannigfaltigkeit]", by distinguishing discrete from continuous manifolds of different dimensions, and aims at identifying the conditions under which a continuous $n$-dimensional manifold admits "measure-determinations [Massbestimmungen]" (Riemann, 1866-1867, p. 138; Riemann, 2016, p. 34), using tools from differential geometry (for a coeval similar approach, see also Helmholtz (1868)), Frege offers, as we will see, a formal definition of an algebraic structure to be understood as the structure of domains of magnitudes, on which real numbers are to be defined as ratios. Whereas Riemann's (and Helmholtz's) perspective is that of a sort of generalized geometry, Frege's is that of an algebraic enquiry, independent of geometry and any other particular theory of specific magnitudes, but able to fix the structural conditions that any domain of such magnitudes is to meet. Far from suggesting making geometry a foundational ground for analysis (which is the position Frege also ascribes to Hankel), or freeing analysis from any connection with geometry (as in the perspective of arithmetization of analysis, which he is openly rejecting), Frege aims at connecting analysis and geometry by means of an independent and separate theory of (domains of) magnitudes, algebraic in nature.

7 The following one, from Frege's discussion of Heine's and Thomae's definitions and their views on "formal arithmetic", is one of them (Frege, 1903, §II.92; Frege, 2013, p. 1012):

Part of the task [of the arithmetician] is [...][to connect] a sense with his formulae; and this sense will then be so general that with the assistance of geometric axioms, and physical and astronomical observations and hypotheses, multiple applications of it can be made in [...] sciences.

This, it seems to me, can be demanded of arithmetic. For otherwise it could happen that this science should treat its formulae merely as groups of figures without sense; and that then, however, a physicist, wanting to make an application of it, might straightaway presuppose, quite without justification, that a thought had been proven to be true. At best an illusion of knowledge would be created. The divide between arithmetical formulae and their applications would not be bridged. For that, it is necessary that the formulae express a sense and that the rules find their grounding in the reference of the signs.

8 Cf. footnote (2), above. 
It is, moreover, doubtless that something similar could also be said concerning his definition of natural numbers. ${ }^{9}$ Still, no clear self-contained formulation of FC is offered either by Dummett and Hale or by Frege himself. The following, suggested by Wright (2000), p. $324,{ }^{10}$ has become the standard reference:

[...] a satisfactory foundation for a mathematical theory must somehow build its applications, actual and potential, into its core-into the content it ascribes to the statements of the theory-rather than merely "patch them on from the outside".

The first thing to be noticed about this formulation is that it presents the constraint as a quite general requirement. The point here is merely that the way in which a mathematical theory is founded should be apt to account for its applications. By a further generalization, one could require that a mathematical theory be so shaped as to make directly clear what it takes for it to be applied. This is certainly a requirement that Frege endorsed. But Frege's point appears to us much more idiosyncratic: it does not generally concern the way a mathematical theory is founded or shaped; it rather concerns the nature which is ascribed to the relevant mathematical objects by defining them in one way or another. Frege had independent reasons and motivations for requiring that arithmetic and real analysis ${ }^{11}$ be conceived as dealing with abstract objects, namely that natural and real numbers should be conceived as such objects. His point was then that the way these objects are defined should directly account for the applicability of these theories. Insofar as, for Frege, defining some abstract objects does in no way coincide with creating them or bringing them into existence, or anything like this, but merely reduces to identifying the relevant objects among those that are there, his point could be expressed as the claim that only those objects whose nature can explain the applications of arithmetic and real analysis could count as the natural and the real numbers, respectively.

§2. The Application Constraint. As anticipated, let us then distinguish a general constraint about mathematical applicability, namely AC—merely requiring, as just said, that the way in which a mathematical theory is shaped be apt to account for its applications - from the distinctive constraint endorsed by Frege, namely FC—-which rather

9 The following passage from Grundlagen could, for instance, be quoted in support (Frege, 1903, $\S 19$; Frege, 1950, p. 26, slightly modified translation):

[...] surely we are entitled to demand of arithmetic that it provide the grounds [Anknüpfungspunkte] for any application made of number [Zahl], even although that application is not itself the business of arithmetic. Even in our everyday sums, we must be able to rely on the science of arithmetic to provide the basis for the methods we use. [...] the number [Zahl] which gives the answer to the question 'How many?' can answer among other things how many units are contained in a length.

10 For other discussions of the way FC should be understood, some of which will be considered later, cf. Hale (2002), Batitsky (2002), Shapiro (2000), Snyder, Samuels, \& Shapiro (2018); and a work in progress by one of the present authors, Sereni (Manuscript).

11 In what follows, we use, for short, the terms 'arithmetic' and 'real analysis' to merely refer to the theories of natural and real numbers, respectively, thus implying (against a frequent use of the former term, often adopted also by Frege, for example in the very title of Grundgesetze), that arithmetic does not deal with real numbers, and avoiding to include the theory of real functions and/or the calculus into real analysis. 
appears to us as a (quite) particular case of such a general constraint. We shall come back later in more details on the latter. For the time being, let us focus on the former.

Let us begin by observing that as general as $A C$ might be conceived to be, it should not be as general as to admit as an instance whatever requirement about the way a mathematical theory is shaped which is concerned with its applicability. In order to count as an instance of $A C$, such a requirement should meet at least two conditions, both of which are motivated by the purpose of being faithful to Frege's views about the applications of mathematics, although generalizing them.

The first condition is this: an instance of AC is to be concerned with applicability, namely with what makes applications possible, rather than with actual applications. A fortiori, no requirement about the way a mathematical theory should be shaped in order to account just for some particular applications, however crucial, should count as an instance of AC. Of course, nothing in principle could disqualify such a requirement, but this would not be in line with the way Frege understands the matter; such requirement could then not plausibly count as an instance of a general pattern of which his constraint, FC, could be seen as a particular case. This is because a requirement like this would be subject to objections paralleling many of Frege's accusations to rival views, both in the case of natural numbers-e.g., Frege's objections to empiricists, who confuse "the applications that can be made of an arithmetical statement [...] with the pure mathematical statement itself" (Frege, 1903, §9; Frege, 1950, p. 13, slightly modified translation; cf. also Frege, 1903, §II.137, footnote 2, on which cf. footnote 13 below.) and in the case of real numbers-e.g., his remark that a definition of them based on "how [geometrical] distances can be determined" is inappropriate for lack of generality (Frege, 1903, §II.75). ${ }^{12}$ It remains, however, that it would be plainly unreasonable to require that any sort of application, even the most fortuitous or occasional, be accounted for by the way a relevant theory is shaped. Natural numbers (or better the symbols habitually used to denote them) are, for example, associated with soccer and other players, in order to quickly identify them during a match. But it would be plainly unreasonable to require that the way arithmetic is shaped be apt to account for this use of natural numbers (or the symbols denoting them). An obvious reason for this is that the same purpose could be achieved with other means, alien to arithmetic. No doubt, Frege was requiring nothing like this. To remain faithful to his conception, one could then only require that the way a mathematical theory is shaped be apt to account for the possibility of certain applications, namely canonical ones, to be taken, in some sense, as more fundamental than others. It follows that the specification of an instance of $A C$, and a fortiori of FC, should depend on the identification of the relevant class of canonical applications.

How such canonical applications should be identified in any case is not easy to see. Steiner (2005), p. 627, has for example suggested, though in a different context, that applications of a mathematical theory should count as canonical "if the theory was developed in the first place to describe" them. Steiner distinguishes between empirical and non-empirical canonical applications, depending on the mathematical theory at hand. Whatever the merits of this suggestion, it is clear that alternative proposals are in the offing. One

12 Literally speaking, Frege rejects such a definition for not being "purely arithmetical". It is however clear that he is here using the adjective 'arithmetical' in a broad sense (cf. footnote 11, above), to qualify a definition of real number depending on the consideration of no independent particular scientific domain. 
may think, for example, that-independently of any specific application the theory was originally conceived to be used for when it was first introduced-different applications should count as canonical if they relate to essential features of the theory itself, i.e., features that only this theory has, or features in absence of which a theory could not count as (a variant of) this very theory. This has a direct bearing on our discussion, for an instance of $\mathrm{AC}$ will be deemed appropriate or desirable only if it requires of a theory that it accounts for applications which qualify as canonical with respect to some methodological or philosophical motivations, which might, in turn, be related to, or equate the very motivations one has for endorsing such an instance of AC. This should appear clearer in what follows.

The second condition concerns the sense in which we could say that a mathematical theory is applied. Not any sense of application is relevant in the context of a discussion of Frege's view. Both Frege and all those taking part in the present debate about his views on the applicability of mathematics are mostly interested in a sort of applicability pertaining to the occurrence of mathematical statements (better: theorems) as premises in arguments whose conclusions concern either some non-mathematical domain or a mathematical domain other than the one to which the relevant theory pertains to. In order for this to happen, the relevant mathematical statements have to be somehow connected with other statements proper to this domain. This connection can take various forms: it can, for example, hinge on the intended meaning of the former statements, to be ascribed to them by the way the relevant theory is shaped and linked with the received meaning of the latter; or it can depend on appropriate bridge principles. In the latter case, the relevant arguments could take the form of appropriate deductions; in the former, they should rather appeal to inferences depending on the relevant meanings, either because of a subjacent formal semantics or, much more frequently, in force of informal considerations or associations.

These simple remarks should be enough to make clear that this second condition is in fact much less restrictive than it might appear at first glance, and indeed much less restrictive that it might be suggested by the label 'semantic applicability' used by Steiner to denote the relevant sense of 'applicability' (Steiner (1998), p. 16; but cf. also Steiner (2005)): all it prescribes is that a requirement counting as an instance of $A C$ be relative to the occurrence of mathematical statements as premises within arguments other than those proper to the very mathematical theory these statements pertain to.

Of course, one could retort that a mathematical theory is never (neatly) separated from a bunch of applications: that the very distinction between pure and applied mathematics is illusory, and that mathematics itself is so intimately unitary as to make it impossible to establish borders among distinct mathematical theories. We don't want to pursue this suggestion here. It will be enough to remember that as worthwhile as such a view might be taken to be, Frege would have not admitted it, so that discussing his views requires assuming that appropriate distinctions can be made between pure mathematics and it applications, mathematical and non-mathematical domains, and a mathematical theory and another to which the former can be applied (crucially between arithmetic or real analysis and geometry or rational mechanics).

The two conditions just discussed are not independent, since one could argue that the canonical applications of a mathematical theory are just those which depend on the occurrence of some (class of) statements of this very theory in arguments concerning some domain stranger to it, possibly to some specified such domain. For example, the case mentioned above, relative to the use of natural numbers (or of the symbols denoting them) to quickly distinguish players in a match, could be taken as a case of non-canonical application, since no arithmetical theorem is involved in it. But, of course, accepting or 
rejecting this option would also depend on endorsing or rejecting some methodological or philosophical motivations, which, once more, could be strictly connected, or even coincide with the very motivations for endorsing one or another instance of AC.

It is, then, relevant, before coming more specifically to FC, to consider which motivations one could have for endorsing $A C$ or some instances of it.

A very broad one might be that meeting $A C$ offers a neat way of keeping together an account of our knowledge of pure mathematics with a suitable epistemology of applied mathematics, and this is something that many (though not everyone) may see as welcomed. Again, and still very broadly, meeting AC might offer a form of no-miracle argument: it could allow arguing that the applicability of mathematics to non-mathematical domains is no lucky accident, but is explained by the intrinsic features of mathematical theories. This also helps locating mathematical knowledge in a general account of knowledge which also includes scientific knowledge.

Again, an appropriate instance of $A C$ might be used, together with other objections, as a further wedge against views on mathematics such as Millian empiricism or some form of formalism (since, according to the two foregoing conditions, it should require, against empiricism, that applicability be explained at a sufficient level of generality and independently of particular applications, and, at least in one of the senses in which the second condition can be conceived, that arithmetical formulae have a meaning, rather than being strings of uninterpreted signs, as a formalism might prescribe). Or it could also be used to lend support to one or another definition of some relevant items. It can help, for example, to decide between a (neo-)logicist account of natural numbers based on Hume's Principle, and a structuralist account based on axiomatic characterization of mathematical structures.

More in general, a platonist taking a mathematical theory to be about a domain of objects which exist prior to this very theory, could appeal to some instance of $A C$, in order to warrant that their prior existence can in fact be posited, since such objects gets defined by resources that do not specifically pertain to this theory and themselves explain why the theory, when suitably characterized, applies to any domain which these resources allow to deal with. A case in point is just that of logicists and neologicists on arithmetic (the case of real analysis is much more puzzling, as we will see in §4), who take it to be about objets defined either by logic alone - this being understood, as Frege repeatedly stresses (cf. in particular Frege (1884), §14), as the most general science, endowed with the utmost applicability (in the sense clarified above)—or, at least, by an extension of logic apt to conserve its applicative generality, or minimize its loss (as neologicists take it to be the case for Frege Arithmetic, the theory obtained by extending second-order logic by adding Hume's Principle to it as a new axiom).

§3. Possible rationales for the Application Constraint. These rather cursory considerations should be enough to make clear that one could have various and quite different motivations for endorsing (different instances of) AC. Delving into some of them in more detail will help us both to better understand (by comparison and immersion in a larger setting) Frege's own motivation for endorsing FC, and to attain the most faithful formulation of such a constraint in relation to the two (quite different) concerns it relates to in Frege's work, namely the definitions of natural and real numbers. At the same time, this will also help understanding how delicate the task is of identifying an appropriate and sufficiently clear instance of $A C$ to be used as a guide in shaping a mathematical theory or in assessing it from a philosophical perspective. 
We will consider and discuss six possible rationales underlying AC. We take an instance $\mathrm{AC}$ to be a general requirement, expressed by a normative statement (either concerned with mathematics as a whole or explicitly specifying its range of application). On the other hand, a rationale for endorsing $A C$ is better seen as a purpose that one could be willing to attain by adopting a certain instance of AC. Advancing or clarifying such purpose does not necessarily require having already specified such particular instance of AC. Typically, things would rather go the other way around: only after that a rationale has been advanced and clarified an instance of AC can be stated at its appropriate level of generality.

Notice that nothing forbids the same instance of $A C$ to be in line with several purposes, that is, that multiple rationales underlie the choice of the same instance of AC. As a matter of fact, this will be the case for the instances of $A C$ we will ascribe to Frege. Notice, also, that some of the rationales we shall consider are outright alien to Frege's (or neo-Fregeans') philosophical views. We will present them nonetheless, both for the sake of completeness and in order to mark the comparison with these views.

3.1. Rationale I: Recovering everyday practice. One first possible rationale for endorsing $\mathrm{AC}$ is the purpose of making (a certain portion of) mathematics as close as possible to our everyday practice, at least for those aspects which somehow pertain to mathematics itself. This would lead one to take as canonical those applications of a mathematical theory (or at least of an elementary and possibly informal core of it) which occur in ordinary contexts or in very basic scientific ones. These will likely involve the use of natural language, although it may happen that some such applications, though inevitably simple in nature, deploy some formal symbolic expressions. Be that as it may, the rationale requires that most common applications of this sort be accounted for in the way the relevant theories are shaped. This can be obtained in different (not necessarily alternative) ways, for example by an appropriate choice of central definitions, or by favoring the establishment of certain relations among the relevant theory and others.

Typically, such a rationale will concern rather basic and/or elementary mathematical theories. But also in these cases, the mentioned criterion for selecting what is to count as canonical applications can appear far from straightforward. Is, for example, mere frequency a sufficient and/or necessary condition for a class of application to be taken as canonical? Which community of users should be taken into account in making a choice? What should actually count as an ordinary context? Moreover, even if an agreement is reached on a selection of canonical applications, their analysis, and, then, the identification of their structure or of the features that the relevant mathematical theory are to account for can also be far from straightforward. Still, a major issue remains also beyond these crucial questions, namely to decide whether the rationale is to be understood descriptively or normatively.

According to the descriptive option, what is to be accounted for by the way the relevant theories are shaped are, if not all, at least the most ordinary applications (assuming one is able to identify some as such).

Against this, it can be retorted that ordinary applications may mislead as regards logical form. Frege (1884), §57, famously argued, for example, that the widespread usage of numerals in adjectival position betrays the correct logical form of numerical statements. Moreover, he suggested that unreflectively paying attention to ordinary uses may lead us to mistake pure mathematical content with particular applications, as Frege alleges Mill to do, for instance, by taking the sign ' + ' to stand for the actual operation of physical aggregation rather than arithmetical sum (Frege, 1884, §9; Frege refers to Schiel's translation of Mill's System of Logic, Bk. III, chap. XIV, §5; this translation counts several editions; possibly 
Frege's reference is to the fourth edition appeared in 1877, translating the eight edition of the original: Mill (1877); Mill (1872)). ${ }^{13}$ Again, mathematical expressions in nonmathematical contexts are often used with a meaning that radically departs from their proper mathematical one, and it would be nonsensical to require that such uses be accounted for by mathematics. To stick to the same example, it would be wholly inappropriate to require that arithmetic should account for the use of 'adding', or to uses of 'and' in idiomatic expressions like 'One litre and one litre make two litres', in which they denote the physical assemblage of, e.g., two liters of water, thus allowing that one plus one may not always be two, as suggested by Mill.

According to the normative option, what is to be accounted for by the way the relevant theories are shaped are, among ordinary applications, those which are taken to respect most faithfully the essential features of the relevant system of mathematical concepts. Some actual applications will be discarded as irrelevant or at best derivative on more fundamental ones, and yet regimentation will aim at preserving those which are seen as pertaining to basic uses of mathematical concepts, as displayed in ordinary discourse.

On this reading, the rationale nicely coheres with a project of conceptual analysis, but is manifestly open to the opposite dangers of vacuity and circularity, unless an intermediate level at which this analysis is to be located is somehow identified: it cannot be analysis of ordinary applications themselves, at the risk of conflating the normative option with the descriptive one; but it cannot be analysis of mathematical theories either, since then the essential features of mathematical concepts to be accounted for will be just dictated by the theories themselves. Absent a general way of identifying such an intermediate level, the problem can be solved case by case via more or less convincing arguments.

Again, Frege's work provides examples. His view that substantival uses of numerals are fundamental over adjectival ones (cf. Frege, 1884, §57), and should be accounted for in a regimented and rigorous analysis, is a neat example of this normative rationale. In line with this, one can submit that we are usually familiar, prior to and independently of our mature arithmetical education with procedures of counting, which, tough quite simple, might be taken as examples of a codified and stable enough (ideal) procedure not affected by the contingence and variability of uses. This might suggest that, if arithmetic is at issue, the relevant conceptual analysis to be invoked by the rationale, under the normative understanding, should be analysis of counting (in one or all of its distinct modalities, such as transitive and intransitive counting: cf. Benacerraf (1965), pp. 49-50, and below, pp. 116-117). ${ }^{14}$

13 Frege (1903, §137, footnote 2), raises similar criticisms to the views advocated by Hermann von Helmoltz in Helmoltz (1887), though making no reference to Helmoltz 's previous essay on "the facts that underlie geometry" (Helmholtz, 1868), where the role of empirical considerations in geometry is also emphasized:

Helmholtz wants to found arithmetic empirically, whether it bends or breaks. So he does not ask: how far can one get without invoking experiential facts? Rather he asks: what is the quickest way I can drag in some facts of sensory experience? Anyone with this aspiration achieves it very easily in the same way, by confounding the applications of the arithmetical propositions with the latter themselves.

14 A possible motivation for endorsing HP somewhat related to the normative reading of Rationale I is discussed by McCallion (2016), p. 318. McCallion argues that 
It is however clear that no similar strategy may be available for less elementary mathematical theories, for no equivalent practice-non-mathematical, yet codified and stable enough to count as an ideal practice not affected by the contingency and variability of uses - can be identified. Real analysis is a case in point: one might think (and Frege was indeed thinking, as we shall see in §4) that measuring stands to it as counting stands to arithmetic; but, when conceived as an everyday practice, measuring is by far less codified and stable than counting, and it is far from manifesting a relation with real numbers as straightforward as that which counting manifests with natural ones (we will come back on this in \$4.1.2). This should be enough to make clear that, even in its normative understanding, the rationale is hostage to a serious limitation. As we will see, a similar objection can be raised also with respect to rationales II and V below.

3.2. Rationale II: Tracking psychological adequacy. Suppose we are presented in practice with a variety of applications of a given branch of mathematics. When asking that an explanation of some of them be accounted for by the way the relevant theories are shaped, we may expect that a given mathematical concept can be supplied to someone lacking it by familiarizing one with such applications. This may be understood merely in a spirit of rational reconstruction. But one may ask for more and require these theories to be also psychologically adequate in some suitable sense, either because they keep track of those elementary applications through which we actually acquire its basic concepts during our psychological development, or because they reflect some biologically entrenched basic mathematical skills. As such, this rationale can be suitable to empirical, cognitively informed reconstructions of mathematics; for instance, it can discriminate between accounts giving cognitive priority to either cardinal or ordinal insights in our mastery of natural numbers, depending on what we take as the most empirically faithful description of our relevant cognitive abilities. However, it may fall short of many philosophical purposes.

To begin with, it may be inapplicable beyond elementary theories, that is for all those portions of mathematics in which the complexity at stake requires highly developed theoretical skills that may not have any direct grounds in our innate biological or neurological make-up, and are not learnt without explicit mathematical training.

Second, a similar rationale is clearly alien to any philosophy of mathematics which endorses Frege's adamant principle "always to separate sharply the psychological from

One of the virtues of Hume's Principle [...] is that it seems to be at least close to something like a conceptual truth with respect to the ordinary concept cardinal number. That's one way in which there can be a close conceptual connection between ordinary arithmetic and the reconstruction based on Hume's Principle, but it's not the only way. We might also consider relationships between concepts, in particular conceptual dependencies. Perhaps the value of abstracting from Hume's Principle lies in the fact that it produces some desirable conceptual dependencies. [...] we might understand rational reconstruction in such a way that the conceptual dependencies amongst our ordinary concepts are taken into account. [...] the more the existing patterns are preserved, the better the rational reconstruction.

A reconstruction of arithmetic via Hume's Principle may fare better than others in so far as the concept 'the number of' (which McCallion considers to be concerned with applications, i.e. with the attribution of cardinality to concepts) "is given a fundamental role: grasp of the concept natural number depends upon a prior grasp [of] the concept the number of". According to McCallion, neo-logicists may motivate reliance on $F C$ on two claims: that "Rational reconstruction requires (maximal) preservation of conceptual dependencies", and that "Conceptual dependencies are (maximally) preserved if and only if the applications constraint is met". 
the logical, the subjective from the objective" (Frege, 1903, Einleitung, p. X; Frege, 1950, p. XXII), his clear statement that "mathematics is not concerned with the nature of our mind, and the answer to any question whatsoever in psychology must be complete indifferent to it" (Frege, 1884, §93; Frege, 1950, p. 105), or his claim that "a [...] description of the internal processes which precede the forming of a number judgment, even the most accurate, can never replace a genuine definition of the concept" since "the number is no more an object of psychology or a product of psychic processes than the North Sea is" (Frege, 1884, §26; Frege, 1950, p. 34). Thirdly, and relatedly, those who endorse such a rationale may be charged of confusing the grasping of thoughts with thinking processes, thought with judging, objective with subjective, and so on.

These objections will be presumably immaterial to those who don't share Frege's antipsychologistic attitude. However, the rationale is clearly inadequate for any constraint that, like FC, is meant to be Fregean in spirit and somehow retrievable from Frege's writings.

3.3. Rationale III: Granting meager applicability. Another possible rationale for endorsing $A C$ is the purpose of getting theories so shaped as to be at least able to provide their central concepts with some context of application, whatever this be. No explicit intent to retrieve common, established, or somehow basic classes of applications from ordinary or scientific practice, nor from cognitive development or biological make-up, would, then, be advanced. One would merely require that these theories be so shaped as to deal with concepts which are not entirely incapable of being applied.

As weak as such a requirement might appear, it may still be seen as essential to block any formalistic approach in Frege's sense (Frege, 1903, §II.86-§II.137): on the assumption that application is conceived in the sense explained above (pp. 104-104), no mathematical theory conceived as Frege's targeted formalists conceive it will be able to explain any application at all, as Frege himself laments (Frege, 1884, §II.91), since such theories would merely deal with meaningless symbols suitable for appropriate manipulation, and not with signs expressing any concept, nor, a fortiori, any concept capable of being applied.

Indubitably, Frege's criticism of formalistic approaches is based on a conception of formalism, and what a formal theory is, which are hardly tenable in light of late developments in (philosophy of) logic. Still, a similar point can also be plausibly made today: one could distinguish between different ways of formalizing a certain portion of mathematics according to their respective capacity of rendering a certain net of concepts. From such a perspective, the requirement, in this first weak reading, would be that a satisfactory formalization should appropriately render those concepts which are involved in one or another application.

This suggests a slightly more substantial reading of the rationale. Suppose we had a previous familiarity with a variety of contexts of application for some informal mathematical notions. In the process of formally rendering these notions, we could select just one or another among these contexts to be accounted for by the relevant (formal) theory.

Despite being in line with Frege's views, in the former reading the rationale appears too weak to provide, in general, any real guidance in selecting, among rival actual formalizations, those having a relevant philosophical significance: for in most cases, all competitor candidates will comply with it. To make just one example, it would be unable to discriminate between rival abstractive definitions. For every abstraction principle will determine at least one context of application: it will be enough to consider how the equivalence relation on its right-hand side partitions the relevant domain in equivalence classes, and the concept introduced will have immediate applications to members of those equivalence 
classes (just like numbers are numbers of, and apply to, equinumerous concepts, directions are directions of, and apply to, parallel lines, and so on). ${ }^{15}$

In the latter reading, however, the rationale appears justifiable only if some reason is provided for the choice of the relevant context of application, which cannot be done without advancing a further rationale. Under this reading, the rationale appears, then, to be either immaterial, or empty, if taken alone.

3.4. Rationale IV: Encoding applicability in the nature of mathematical objects. A much stronger rationale for endorsing $A C$ consists in requiring that the applicability of a mathematical theory be somehow accounted for by the very nature of the mathematical objects this theory deals with.

This rationale relies, of course, on the presupposition that mathematics deals with objects. Usually, these will be taken to be both abstract and mind- and language-independent. Beyond this, however, the presupposition is neutral as to how these objects are to be conceived. We can thus distinguish two readings of it.

On one reading - the restrictive reading, as we shall call it - the presupposition includes the assumption that these mathematical objects have an intrinsic nature, a proper haecceity, which is given by (some of) their individual, non-relational properties. This is the conception usually associated with platonist views à la Frege.

On the second, more relaxed reading - the liberal reading, as we shall call it - the presupposition is, instead, uncommitted to any specific feature of mathematical objects; it merely consists in granting that mathematics deals with objects, and is thus also consistent with the idea that mathematical objects are merely specified by a set of relational properties, or more generally by a number of conditions the relevant mathematical theory is required to establish. Moreover, it is consistent with the idea that the objects a mathematical theory deals with are not individual objects (e.g., natural numbers), but structural objects (e.g., the abstract structure of progression). On this reading, the rationale is consistent with structuralist views like ante rem structuralism (Shapiro, 1997).

In the former reading, the rationale nicely coheres with Frege's conception of numbers, both natural and real, as it is displayed, for example, when he laments that in some rival definitions of real numbers applicability lacks "any internal connection grounded in the nature of the number itself” (Frege, 1903, §II.159; Frege, 2013, p. 1572).

Beyond Fregean exegesis, the rationale, even in its liberal reading, may however be charged of being too strong or biased. Under its restrictive reading, it will only support instances of $A C$ which can be endorsed by those who share its presupposition, so that any understanding of a mathematical theory as not dealing with (abstract) objects endowed with a proper haecceity will be immediately ruled out. Under the liberal reading, it will also support instances of $A C$ that could be endorsed by those who take mathematics to deal with structural objects, but it would remain incompatible with non-platonist views, denying that mathematics deals with (abstract) objects of any sort at all. Although we

15 Thanks to Roy Cook for drawing attention to this point in conversation. Notice that there is clearly another very minimal sense of applicability which is delivered by the case of simple predication: a concept $\mathfrak{F}$ is applicable in this minimal sense if it is possible, given an entity of the appropriate level $\alpha$, to establish whether it is or it is not the case that $\mathfrak{F}(\alpha)$. On this minimal sense, any concept (be it sortal or not) for which such conditions of application are given will turn out as applicable, i.e., arguably any concept which is not blatantly defective. Therefore, any definition of a non-blatantly defective concept will determine at least one basic context of application, given by simple predication. Clearly, here we have in mind applications that go beyond this minimal sense of applicability. 
will not explore the issue here, it may be argued that versions of $A C$ which are backed by either readings of this rationale are unwarranted exactly in so far as their underlying metaphysical presupposition bans a vast number of philosophical accounts of mathematics from the start. ${ }^{16}$

3.5. Rationale V: Mirroring concept formation. Yet another rationale is suggested by the following remark by Wright (2000), pp. 328-329:

Frege's Constraint ${ }^{17}$ is justified, it seems to me, when — and I am tempted to say, only when-we are concerned to reconstruct a branch of mathematics at least some-if only a very basic core-of whose distinctive concepts can be communicated just by explaining their empirical applications.

This suggests that one motivation for endorsing $A C$ could be the purpose of fashioning some (presumably elementary) mathematical theories so as to directly reflect those applications which are either paradigmatically employed in conveying some basic concepts involved in these very theories to those who lack them, or are, at least, such that no proper understanding of these concepts could be obtained without reflecting on them. Such a rationale would, then, motivate instances of $A C$ requiring that a mathematical theory be shaped so as to account for applications that-in so far as they are capable of introducing the relevant mathematical concepts-are available or familiar before any explicit mathematical theorizing, being rather what such theorizing is meant to reconstruct and systematize.

As Wright's passage suggests, this rationale would not be appropriate with respect to any mathematical theory, since it would be quite odd to suppose, let alone require, that any such theory involve concepts which are either paradigmatically conveyed by some of their applications, or even only understandable by looking at these applications. Indeed, Wright makes his remark in order to show that is just not the case for real analysis (more on this in $§ 4.1 .3$ ).

This rationale should not be conflated with Rationale II. The latter concerns the way a mathematical theory should be shaped in relation to our psychological processes or cognitive abilities; the former concerns the way such a theory should be shaped in relation to the very nature of the relevant concepts. In the former case the focus is on psychological adequacy and cognitive skills, up to possibly suggest a (psychologically or cognitively) appropriate choice of concepts in shaping the relevant mathematical theories; in the latter, the focus is on the relations that some mathematical concepts (which are taken to be) intrinsically involved in these theories (are taken to) have with some of their applications.

Also this rationale can be given two different readings. One restrictive reading follows Wright in focusing on empirical applications. As will be discussed at more length in $\S 4.1 .3$, this is what motivates Wright's belief that AC is justified in the case of naturals but not in the case of reals. For while there may clearly be basic applications of natural numbers in counting empirical collections which can be familiar before any full-fledged mastery of

16 An exploration of instances of $A C$ which are supposed to escape this charge is offered in Sereni (Manuscript).

17 Remember that Wright employs the term 'Frege's Constraint' to refer to the requirement stated in the passage quoted above, p. 102, namely to what we labeled 'AC'. 
arithmetic, there are no such applications of real numbers available in simple empirical contexts before (at least partial) mastery of a systematic theory of real numbers.

However, on a more liberal reading, one may rather focus on basic extra-theoretical applications generally, be they empirical or not. For instance, one may take the apprehension of the very notion of a real number to hinge on some relatively simple geometrical constructions. A similar attitude seems to be in line with Frege's views, since, in speaking of applications of real analysis he seems to be thinking also (if not generally: we will come back on this in $\$ 4.3$ and $\$ 4.2$ ) to geometrical applications. The passages quoted above (pp. 99-100), respectively, taken from §II.75 and §II.159 of the Grundgesetze, are explicit on this respect.

In this liberal reading, the rationale seems then to be able to support instances of $A C$ suitable for both theories of natural and real numbers, and arguably for any mathematical theory, since applicability of mathematics on itself is not only pervasive, but also distinctive of it.

3.6. Rationale VI: Tracking the architecture of mathematics. An entire chapter of Gandon's book on Russell's (and Whitehead's) treatment of geometry and the "theory of quantity" in The Principles of Mathematics (Russell, 1903) and Principia Mathematica (Russell \& Whitehead, 1913), is devoted to the role of AC in the latter book, namely what Gandon calls its "architectonic use" (Gandon, 2012, chap. 6, especially §6.3). Gandon’s basic point is that $A C$ is not intended there "as a substantive demand for connecting two bodies of knowledge [...] which are conceived as distinct", but rather "as a tool for delineating the outlines of a concept or theory, whose content is not considered as already fixed" (Gandon, 2012, pp. 12-13). Here is how Gandon explains the point:

Far from being regarded as a given datum, the division of the mathematical field into well-defined, distinct branches is a task that the logical analysis is supposed to perform. And [...][AC] is instrumental in the fulfillment of this work: to look at different contexts of use is a means of probing different ways of carving out the mathematical content and to make definite the borders of the different disciplines. There is thus a feedback effect in the [...] use of $[\ldots][\mathrm{AC}]$ : the examination of the applications of a particular concept gives information on its content. Russell and Whitehead did not see [...][AC] as a principle allowing them to relate two already separated fields of knowledge, but as a device enabling them to locate the joints at which mathematics must be carved.

This might be seen as being in contrast with the second condition for $\mathrm{AC}$ expounded in $\S 2$, in particular with the acknowledgement of a clear-cut divide between distinct mathematical theories. But it is not, in fact. For the point, here, is not that Russell and Whitehead denied such a divide, but that they did not take it as given prior to their reconstructive work, and rather conceived it as an outcome of such work.

This suggests a new rationale for endorsing AC, namely using it as a guide for appropriately connecting mathematical theories either to each other or with some non-mathematical ones, by shaping them accordingly. The aim, here, is less that of letting these theories deliver a picture of mathematics in line with some general ontological, epistemological or metaphysical views, than that of organizing mathematics in a way that corresponds to a suitable geography of knowledge.

It may come as a surprise that we consider here the case of possible connections between mathematical and non-mathematical theories. Apparently, this is not a case Gandon was 
thinking of, when advancing his remark. Admitting this possibility is however crucial in order to take this rationale as relevant for our discussion of FC in relation to real numbers. As we have said below, Frege's basic idea was to define reals as ratios of magnitudes, namely as measures of them. In $\S 4.3$, we will contend that taking such a definition to comply with (an instance of) FC is tantamount to subscribing to this rationale. But this could not be the case if connections with non-mathematical theories were left out. The reason, briefly, is the following (we will come back on this in §4.2).

To the purpose of offering that definition, Frege previously offered a formal definition of domains of magnitudes, ${ }^{18}$ which can be viewed as a way of establishing a formal theory of such domains. Defining real numbers as ratios of magnitudes would have, then, ipso facto led to connect real analysis to this theory. Arguably, this latter theory should count as a non-empirical theory, but it should also be able to reflect the relevant structural features of any particular domain of magnitudes, either mathematical magnitudes, like geometrical ones (including segments or curves length, surfaces area, solids volume, angles amplitude, or non-(purely)-mathematical ones, like length (of physical bodies), mass, light intensity, electric charge, etc. ${ }^{19}$ Hence, connecting real analysis to a formal theory of domains of magnitudes would have the effect of connecting it to other theories too, both mathematical (namely geometry or its relevant branches), and non-mathematical ones.

3.7. The many roles of the Application Constraint. The foregoing rationales are not meant to be mutually exclusive. Nor they are required to be satisfied conjunctively. Nor it is the case that a single instance of $A C$, satisfying one or more of these rationales, should apply across the board to definitions of any mathematical theory. Several combinations are therefore possible. A given instance of AC may comply with more than one rationale. Or it can comply with one of them, and disregard others. Finally, it may happen that some instance of $\mathrm{AC}$ (complying with some but not necessarily all of rationales I-VI) inform some mathematical theories, while other instances inform other theories.

In what follows, we'll consider how these different rationales may be at play in Frege's reflections on applications and applicability, in relation to definitions of both natural and real numbers.

§4. How to interpret Frege's Constraint. The rationales discussed in the previous section fit with AC in its full generality; as such, the instances of $A C$ they underwrite do not force any particular way of shaping the relevant theories.

Only Rationale IV is a little more specific in this regard, since it compels the relevant instances of $A C$ to grant that the relevant theories deal with abstract objects. Even though this primarily concerns the way these theories are understood-since the very same theory can be taken, from different philosophical perspectives, either to deal with abstract objects

18 Cf. footnote (2), above.

19 Here is what Frege writes in discussing Cantor's definition of real numbers (Frege, 1903, §II.73; Frege, 2013, p. 842, §II.73):

What then is the substance of the assertion that number-signs designate quantities? Let us look at the applications of arithmetical laws in geometry, astronomy, physics. Here, indeed, numbers occur in relation to magnitudes, such as length, mass, light intensity, electric charge [...]. 
or not-it remains that it would be hard to admit that a theory deals with objects if it involves no (explicit or implicit) definition of (a domain of) first-order items. A quite natural way of conforming with this rationale is, then, to endorse instances of $A C$ advancing a specific requirement on the definitions involved the relevant theories, namely that at least some of them define first-order items, and that in doing so they ascribe to these items an appropriate (intrinsic or relational) nature. Since there is little doubt to us that Frege understood FC (or the different instances of it that he endorsed) in exactly this way (i.e., as a principle imposing specific constraints on how definitions should reflect the nature of first-order items), we can safely assume that the instances of $A C$ which may be suggested by Rationale IV will conform to Frege's way of conceiving AC.

We can, then, take FC to be a general pattern for those instances of $A C$ which-by complying with Rationale IV in one of its readings (or in both) - take it for granted that the relevant theories include definitions of first-order items, liable to be understood as the (presumably abstract) objects this theory is about, and advance requirements relative to these very definitions. This leads to the following general formulation of FC:

[FC] Suitable definitions of the mathematical objects (or first-order items) a mathematical theory is about must be such that its canonical applications be built into the very nature that these definitions ascribe to these objects, that is, into features of them which are immediately manifested in the definitions themselves.

We take an instance of FC to be any constraint that specifies this general requirement one way or another.

It is certainly possible to specify this general constraint in ways which Frege would not have accepted. For example, it is possible to imagine instances of this general constraint which comply with the liberal reading of Rationale IV but license definitions of mathematical objects as objects which only possess a relational or structural nature; clearly, this would be alien to Frege's view. However, this has to do more with the way in which any particular instance is specified, than with its being an instance of this general requirement. Therefore, there appears to be no further room for specifying FC as a particular case of $\mathrm{AC}$ just on considerations concerning its form. The fact that any version of FC complies with Rationale IV does in no way entail that an instance FC could not also comply with any other of the rationale above. The rationales, by themselves, are neutral as to whether an instance of $\mathrm{AC}$ complying with them should also count as an instance of FC. It follows that different instances of FC can be endorsed depending on which among the rationales I-IV one is driven by. In the present section, we will explore this matter with respect to Frege's views.

4.1. Semantic Frege's Constraint. By itself, FC does not presuppose that there are applications of a mathematical theory, in particular canonical ones, that can be identified independently of the theory itself, or independently of the theory when it is so shaped as to agree with such constraint. For FC to be a satisfactory requirement, all it takes is that one can look at the theory, shaped in a certain way, and identify its canonical applications (applications which can also be made available once the theory itself is formulated, or by the fact that it is shaped in a certain way), and evaluate whether what explains these applications is or is not built into the definitions used in the theory as shaped in that particular way.

A theory, however, may happen to be somehow available even before being shaped in some particular way. It may for instance be available as an informal theory which is used 
in practice even before a proper formalization is advanced. Or it may be a formal theory which nonetheless admits of alternative formalizations. This is actually a typical situation which foundational projects in the foundation of mathematics are confronted with. To make the simplest example, there is no doubt that arithmetic can be taken as given and, as such, can have practical applications, also independently of whatever formalization of it, or, a fortiori, of this or that formalization. In such cases, the canonical applications of the theory may be identified independently of any particular way of shaping it, as those applications which are available for the theory even before it is shaped in that particular way. When referring to this case, we will say that these applications are inherent to the theory.

But it can also happen that these applications have (or acquire, with time and practice) a sort of autonomy from the theory itself, however it is shaped. They employ some elements of the vocabulary proper to this theory, or of one of the ways it is fashioned (typically terms, predicates or functors), but they do so in the framework of some codified practice which has the effect of letting such elements acquire a meaning independently of the theory itself, in any of its ways of being shaped, or at least independently of its hard core (assuming this has somehow been identified). Let us, then, say that this sort of applications are external to the theory. ${ }^{20}$

Supposing, as said above, that a mathematical theory include definitions of first-order items, liable to be understood as the objects this theory is about, one could then require that these definitions identify these objects as those which these external applications appeal to (although accessing to them in a different way).

Such a requirement would, however, be in order only if one also granted that the relevant external applications actually appeal to objects that a mathematical theory can be about, presumably (or better, certainly, in a Fregean setting) abstract ones. One might judge this condition too strong, since even a platonist arguing that the relevant mathematical theory deals with self-standing objects could plausibly refrain from taking this to be the case also for its external applications. This suggests weakening the requirement by simply demanding that the relevant definitions ascribe, to the terms denoting the objects they define and to the predicate designating the property of being one of them, a meaning which is apt to recover, within the theory they belong to, the meaning they have when used in these applications. Provided that these applications be taken as canonical, one would get, then, a possible specification of FC.

Under the instances depending on this specification, $\mathrm{FC}$ is a semantic constraint. Call, then 'Semantic FC' ('SFC'), the general pattern these instances obey to. In them, the relevant definitions are meant to reflect the use that is made of the relevant terms and predicates in the external applications of the relevant theory, if there are any which count as canonical.

Whether a certain mathematical theory might be taken to have some external applications or not is however outside the range of the requirements an instance of AC (and $a$ fortiori of FC) can advance: it depends on facts and conceptions which are independent of any choice that $A C$ can suggest or be a guide for. If this mathematical theory is taken to have external applications, then an instance of SFC concerned with it can require these applications to count as canonical. But if this mathematical theory is not taken to have

20 In $\$ 4.1 .1$ and $\S 4.1 .2$ a case will be made for the possibility of conceiving some applications both of arithmetic and real analysis as external ones. This will involve examples that should help to better clarify what we mean by external applications of a mathematical theory. 
such applications, any putative instance of SFC concerned with it would be completely pointless, and the same will hold for any putative instance of SFC concerned with a theory which were taken to have external applications, which wouldn't nonetheless be identified as its canonical ones. No such instance of SFC could, then, comply with any of the rationales presented above. We shall then consider a requirement akin to SFC to be well stated only if it is concerned with a theory which is taken to have external applications and if it identifies these applications as the canonical ones.

The very fact that the canonical applications of a certain mathematical theory, considered in an instance of SFC, are (taken to be) external suggests they could be just those which occur in ordinary contexts or in very basic scientific ones. If this is so, the relevant instance of SFC does not only comply with Rationale IV in one its readings (or in both), but also with Rationale I, in its descriptive reading, at least. Though not significantly, indeed, any instance of it will also comply with Rationale III. Its complying or not with rationales II, $\mathrm{V}$ and VI depends, instead, on further, more specific factors.

The question we are mainly interested with is whether some instances of SFC can be ascribed to Frege. It is clear that, for it to be the case, such instances are to comply with Rationale IV in its restrictive reading. If we take this to be the case, we get a different answer in the case of natural and real numbers. Let us consider these two cases at once.

4.1.1. Semantic Frege's Constraint for natural numbers. A quite natural instance of SFC concerning (the theory of) natural numbers and complying with Rationale IV in its restrictive reading could be the following:

[SFC $\left.\left(\mathrm{Nat}_{\mathrm{tc}}\right)\right]$ Suitable definitions of natural numbers, as objects arithmetic is about, must be such as to ascribe an intrinsic nature to each of these numbers, and do it in such a way that the applications of arithmetic pertaining to transitive counting be built into this very nature in so far as these applications are external, and the meaning both of numerals and of the predicate designating the property of being such a number (respectively as terms and a predicate used within arithmetic itself) be apt to recover, within (the relevant version of) arithmetic, the meaning these very terms and this predicate acquire when used in these (external) applications.

Here the presupposition is made both that applications of arithmetic pertaining to transitive counting are external and count as canonical, and that their outcome is correctly expressed using (the same) numerals (as those at work in arithmetic). This might be debatable. But the point here is not whether this presupposition is correct, but rather whether it fits with Frege conceptions.

This largely depends on how transitive counting and arithmetical applications pertaining to it are conceived of. Benecerraf takes transitive counting to consist in counting "the elements of some $k$-membered set $b$ [...][by establishing] a one-to-one correspondence between the elements of $b$ and the elements of [...][the set of the natural numbers] less than or equal to $k$ " (Benacerraf, 1965, p. 50). Taken literally, this suggests that performing transitive counting depends on mastering relatively advanced mathematical notions, which would entail that no application of arithmetic pertaining to it could count as external. Still, less demanding accounts are possible: in particular, accounts which make transitive counting depend on mastering no arithmetical notion at all.

Consider a cognitive subject Lalo which is able to distinguish some items (possibly objects) from each other, and to delimit them, so as to also distinguish them from any other 
item. Suppose also that Lalo is able to take advantage of these abilities in considering these items one at a time, by also keeping track of those already considered. These seem to be abilities a cognitive subject must have to perform any form of transitive counting, though possibly still not enough for making one able to perform even a rather rudimentary form of it. But if Lalo did proceed this way alternatively on two distinct lots of items (namely, by considering an item of one lot, then an item of the other lot, then coming back to the former lot and considering an other item of it, and so forth), by also being able to evaluate whether the two lots run out at the same stage, or one of them runs out when the other still does not, it might be in order to take him as being actually performing transitive counting. Unless one of the lots were given by an initial segments of numerals, taken in their order, no resource somehow pertaining to arithmetic would be at work here, and it would hard to take this as an application of it. But if we suppose instead that the elements in one lot are indeed numerals, taken in their order, one could take this procedure as an application of arithmetic, and presumably as an external one, since, within a practice like this, the relevant numerals would acquire a meaning that only depends on their being considered at a certain stage of the procedure, and is then wholly independent of arithmetic itself, in any of its ways of being shaped. The order of numerals is, indeed, as such, purely lexicographic, depending on their compositional properties and relations (which are in no way proper to natural numbers themselves), and is, then, merely fixed by a number of appropriate linguistic rules. Lalo's familiarity with these rules, and his consequent capacity of uttering numerals in their order up to the end of the procedure, for whatever given other lot of items, would make him able to associate a numeral to such a lot (that uttered at the last stage of the procedure), while his capacity to repeat the procedure in a number of other relevant circumstances would make him acquire, from this association, a quite useful information for many practical purposes. Were him able, for example, to repeat the procedure both on a lot of oranges, to each of which a price of one dollar is attributed, and to a wad of $1 \$$ dollar notes, he could be able to buy the oranges and pay the right prices. His further capacity of compositing numerals by addition and multiplication (as defined and conceived as operations on numerals themselves) would not essentially change the situation. Had him also this further capacity, he could use numerals in most of the usual ways they are used in our every-day life and generally conceived as depending on transitive counting, still without ascribing to them a meaning perfectly independently of (any version of) arithmetic itself. A way to support this last point could be by observing that Lalo could act in the way just described without having any idea of the infinity of numbers, or even of numerals, and even by being perfectly unaware that they could be used to name non-compositional items forming a progression, namely natural numbers.

So conceiving transitive counting makes it plausible to take it not only as pertaining to external applications of arithmetics, but also as the very source from which elementary arithmetical notions come from, and then to take SFC( $\left.\mathrm{Nat}_{\mathrm{tc}}\right)$ not only as well stated, but also as complying, not only with Rationale IV and, presumably, Rationale I in its descriptive reading, but also with Rationale V, and, possibly, Rationale I, in its normative reading, too.

There is no direct evidence suggesting that Frege would have conceived transitive counting this way, but it seems clear that, under this conception, SFC $\left(\mathrm{Nat}_{\mathrm{tc}}\right)$ comes to require that arithmetic include definitions of natural numbers and of the predicate designating the property of being such a number which conform to a conception of these numbers as (abstract) objects associated with collections of items in such a way that the same cardinal is associated with two such collections if and only if a bijective relations can be defined 
among the items in these collections. In Frege's views, the relevant collections of items should be taken as those formed by the objects falling under (first-level) concepts (or, if you prefer, as the extension of these concepts), so that this requirement becomes that of defining natural numbers as cardinals of finite (first-level) sortal concepts, so as to provide a measure of their cardinality conforming with Hume's Principle. This is perfectly in line both with his basic claim that "a statement of numbers contains an assertion about a concept" (Frege, $1903, \S 46, \S 55, \S 57$; Frege, 1950, pp. 59, 67-68, slightly modified translation), and with his well-known definition of natural numbers (Frege, 1903, §68, §74, §77, §83; Frege, 1903, §I.41-§I.42, §I.46), and his proof of Hume's Principle from this definition (Frege, 1884, §73; Frege, 1903, Theorems 32 and 49). This suggests that, in so far as arithmetical applications pertaining to transitive counting are conceived of as just said, Frege actually endorsed SFC( $\left.\mathrm{Nat}_{\mathrm{tc}}\right)$, according to a conception of transitive counting close to the one expounded above, and, even, that this is just the instance of FC which is actually at work in Frege's definition of natural numbers.

In the Grundlagen, Frege mentions an alleged geometrical definition of numbers, including natural ones, which he ascribes to Newton, then he makes the following remark (Frege, 1884, §19; Frege, 1950, p. 26):

[...] we should [...] remain in doubt as to how the number defined geometrically $[\ldots]$ is related to the number of ordinary life, which would then be entirely cut off from science. Yet surely we are entitled to demand of arithmetic that its numbers should be adapted for use in every application made of numbers, even although that application is not itself the business of arithmetic. Even in our everyday sums, we must be able to rely on the science of arithmetic to provide the basis for the methods we use.

Here Frege seems to take "ordinary life" to involve "applications [...] of number" as defined in arithmetic. It is still quite doubtful that one could take the everyday-life use of numerals to depend on a conscious application of whatever version of arithmetic, understood as a genuine mathematical theory. Requiring that arithmetic define natural numbers so as to make them "adapted for use" in "ordinary life" seems much more plausibly the same as requiring that its definitions conform to an independent use of numerals, which is in agreement both with SFC( $\left.\mathrm{Nat}_{\mathrm{tc}}\right)$ and with the foregoing account of transitive counting. This is the way we suggest this passage is to be understood.

In Frege's view, logic provides a general theory of sortal concepts and objects. Hence, aiming at defining natural numbers as objects associated with finite such concepts naturally leads, in his view, to trying to define them within logic, and then to intimately connecting arithmetic to it. There is, then, room to argue that Frege's foundational program just hinges on this, namely that it was not logicism which led him to his conception of natural numbers as cardinal numbers and to the corresponding definition of them, but, rather, in the other way around, his conception of natural numbers as cardinal numbers, driven by SFC(Nat $\left.t_{\mathrm{tc}}\right)$, which led him to try to get arithmetic within a system of logic. If this is granted, SFC(Nat ${ }_{t c}$ ) might also be taken to comply with Rationale VI (but on this, later).

4.1.2. Is there an instance of the semantic Frege's Constraint for real numbers? Once it is admitted that Frege's definition of natural numbers obeys to SFC $\left(\mathrm{Nat}_{\mathrm{tc}}\right)$ as the relevant instance of FC, a natural question arises: would Frege's envisaged definition of real numbers have obeyed to some other instance of SFC? 
If the answer were openly positive, we would be entitled to submit that FC plays a role in Frege's foundational program under the form of a semantic requirement, namely SFC. A negative or more nuanced answer would rather suggest that Frege did not conceive of FC in the same way in relation to the tasks of defining natural and real numbers, respectively. Moreover, if one also admits, as we think one should do, in agreement with Dummett and Hale, that Frege did not merely conceive FC as a pertinent requirement his definitions of both sorts of numbers should better meet than don't, but rather as a crucial, if not essential one, namely as a mandatory guide for these definitions, such an answer might even have consequences on the way Frege's foundational program is understood.

In the present subsection we will argue in favor of a quite nuanced, if not negative answer. In next $\S 4.3$ we will suggest an alternative way to specify FC so as to make Frege's envisaged definition of real numbers comply with it. Finally, in $\$ 5$ we will consider which consequence this might have on the understanding of Frege's foundational program.

If FC actually played a role in orienting Frege's ideas about the way real numbers should have been defined, the relevant canonical applications would certainly concern the measure of magnitudes. Hence, if an instance of SFC provided a guide for his envisaged definition of these numbers, it would be as follows:

[SFC $\left(\right.$ Real $\left.\left._{\mathrm{mm}}\right)\right]$ Suitable definitions of real numbers, as objects real analysis is about, must be such as to ascribe an intrinsic nature to (each of ${ }^{21}$ these numbers, and do it in such a way that the applications of real analysis pertaining to the measurement of magnitudes be built into this very nature in so far as these applications are external, and the meaning both of numerical terms purportedly referring to these numbers and of the predicate designating the property of being such a number (respectively as terms and a predicate used within real analysis itself) be apt to recover, within (the relevant version of) real analysis, the meaning these very terms and this predicate acquire when used in these (external) applications.

The problem is whether this could count as an instance of SFC or should rather be taken to be ill-stated. Doubtless, real analysis has inherent applications pertaining to measurement of magnitudes. But is there room for taking these applications also as external ones, and, then, for claiming that the numerical terms used within this theory to purportedly refer to real numbers (such as ' $\sqrt{2}$ ', ' $\pi$ ', but also ' $\frac{2}{3}$ ' or ' 3 ', if used to name real numbers, rather than merely rational or natural ones) and the predicate used there to designate the property of being such a number can, in these applications, acquire a meaning independently of this very theory, in any of its ways of being shaped?

To answer this question, let us begin by wondering what should one take a magnitude to be when speaking of applications of real analysis pertaining to the measurement of magnitudes.

One could think Frege could have answered that it should be an element of a domain of magnitudes as he himself defined it. As we know, his definition depends on an inconsistent theory, and this could be taken to be enough for rejecting this answer. We also know,

21 One could doubt that it be reasonable to demand that each item among non-countable many ones could be endowed with an intrinsic nature, and even doubt that Frege would have required this. This is a difficult question which we neither want nor need to address here. 
nonetheless, how to amend this definition so as to make it immune from inconsistency. ${ }^{22}$ We could, then, easily offer a modified answer by taking a magnitude to be an element of a domain of magnitudes as it is defined by a consistent rephrasing of Frege's definition.

This consistent rephrasing certainly involves essential ingredients also involved in an appropriate consistent rephrasing of Frege's envisaged definition of real numbers. However, the former would be, as such, perfectly independent of the latter. At most it would be a rephrasing of Frege's envisaged proof of existence of appropriate domains of magnitudes that would depend on the definition (or construction) of a domain of items (defined on the basis of natural numbers) which share the same structure as that of the real numbers. ${ }^{23}$ Such consistent rephrasing of Frege's definition of domain of magnitudes could, then, be taken as a way to establish a general theory of magnitudes independent of real analysis, and so apt to also identify magnitudes, at least structurally, independently of real numbers. Definitions of (domains of) magnitudes similar to Frege's (although consistent) have been advanced by others in different ways, and different occasions. ${ }^{24}$

Getting such a general theory of magnitudes, and the ensuing (structural) identification of magnitudes leaves, however, the problem open of explaining how magnitudes so defined could be measured. Frege's envisaged solution depends on ipso facto identifying their measure with real numbers, just defined as ratios on them, which is in open contrast with the very idea that the applications of a theory of real numbers to the measurement of magnitudes could be external, since it makes it in principle impossible to speak of measure of magnitudes without invoking this very theory. Are there other strategies that can be followed to solve the problem independently of any appeal to this very theory, and thus to foreshadow some sort of external applications of it? This seems quite doubtful, unless one appeals to unnatural and unnecessary duplicates of real numbers (which, by the way, would differ from them only nominally, but not structurally).

This is an obvious consequence of the fact that any general theory of magnitudes whatsoever, be it in line or not with Frege's, cannot but be structural (that is, merely depending on fixing a structure). According to Frege's definition of domains of magnitudes, these are identified with value-ranges of permutations satisfying some appropriate structural conditions. For the sake of consistency, such value-ranges should be replaced with those permutations themselves. But permutations on what? If one wants to identify magnitudes as the subject-matter of some putative external applications of real analysis, not depending on structurally indistinguishable duplicates of these numbers, one needs to answer to this last question. Still, this is something that neither Frege's original definition, nor any consistent rendering of it can make, simply because of its being a structural definition. And, mutatis mutandis the same holds for any other definition depending on a general (and, then, necessarily structural) theory of magnitudes.

One could argue that there is another way of establishing what should a magnitude be taken to be, in the context of an enquiry about the nature of the applications of real analysis

22 A way of doing it within a system of third-order logic is suggested in a co-authored work in progress by one of the present authors, cf. Boccuni \& Panza (Manuscript).

23 Cf. again the work mentioned in footnote (22), above.

24 O. Hölder advanced, for instance, such a theory two years before the second volume of the Grundgesetze (Hölder, 1901; Dummett, 1991, pp. 281-283), and a much more recent alternative (rendering, in a modern setting, the basic ideas of Eudoxus-Euclid's theory of proportion) has been advanced by D. Scott in an unpublished document, mentioned and summarized in Peacocke (2015, pp. 371-374), where a "straightforwardly realistic attitude to the ontology of magnitudes" (ibidem, p. 362) is also argued for. 
to measurement of magnitudes, which is still in line with Frege's definition of domains of the latter. It would simply consist in taking a magnitude to be an element of whatever system which exemplifies the structure Frege attributes to such domains. One could argue that this answer would parallel that which should be given, in Frege's spirit, if it were asked what it is that one should take to be counted in transitive counting when speaking of applications of arithmetic pertaining to it. As observed above, the answer would be that it counts objects falling under sortal concepts.

There is, however, an essential difference among these two answers, which is made manifest by the fact that the latter is directly understandable by anyone who is able to understand the basic notions on which Frege's system of logic is grounded, whereas understanding the former requires understanding a quite convoluted (structural) definition. True: this definition can be performed within a quite weak system of third-order logic (so weak as to include only second-order comprehension). ${ }^{25}$ However, whereas understanding this definition, and then having a clear idea of what a magnitude is, requires being familiar with this very system of logic, no familiarity with any system of logic is required to have a clear idea of what transitive counting counts, in general, even if we accept Frege's idea that it counts objects falling under sortal concepts. The general notions of an object and a sortal concept ground our understanding of Frege's system of logic (and, possibly of any modern system of higher-order logic), not the other way around (if this were not the case, the applications of arithmetic pertaining to transitive counting would not be external, and SFC $\left(\mathrm{Nat}_{\mathrm{tc}}\right)$ would be ill-stated and could not count as the instance of SFC that is at work in Frege's definition of natural numbers).

The situation, thus, seems to be significantly different from what happens for the canonical applications of arithmetic, as depicted in SFC(Nat tc $_{\text {c }}$, and for Frege's definition of natural numbers. For here the very identification of the subject-matter of the canonical applications of real analysis, as depicted in SFC(Real $\mathrm{mm})$, depends, in Frege's spirit, on essential ingredients involved in his envisaged definition of real numbers.

Though symptomatic, this difference has, as such, no direct consequence on the question of deciding if such applications are external. The reason is, once again, that, although Frege's definition of domains of magnitudes involves essential ingredients which are also involved in his envisaged definition of real numbers, the former is intrinsically perfectly independent of the latter. It remains, however, that taking a magnitude to be an element of a system exemplifying the structure of domains of magnitudes is by itself not enough for identifying a way of measuring the elements of such a system which does not depend on the availability of structurally indistinguishable duplicates of real numbers, if such a system is not explicitly identified.

This suggests an alternative strategy, namely arguing that the identification of the subjectmatter of putative external applications of real analysis pertaining to measurement of magnitudes depends on specific inquiries, typically empirical, geometrical or mechanical (in the sense of being proper to rational mechanics, conceived as a branch of mathematics). This would make the very explanation of what counts as such an application also hinge on these specific enquiries, which could not but be quite different to each other. Though this would be openly alien to Frege's thought, inspecting this strategy will be instructive.

Whatever the relevant enquiries might be, they should be adequate to fix identity conditions for the magnitudes they are concerned with. And, insofar as what is here in question is a sort of measurement which should be apt to be rendered as measurement by real

25 Cf. the work mentioned in footnote (22), above. 
numbers, these conditions should be such that $a$ is the same magnitude as $b$ only if the same real number measures both $a$ and $b$. But suppose that a magnitude identified though an empirical enquiry is measured by empirical means. There would be no way to determine a single real number which measures it, since, for any such number $r$ which one could take as the result of an empirical measurement of such a magnitude, there would be a neighborhood of $r$ (which would vary with the methods and conditions of measurement, but would be always nonnull), such that it would impossible to discriminate, empirically, wether the result of such a measurement is actually $r$ or any other real number included in such a neighborhood.

Another, more general, way to tell the story is this: no domain formed by items empirically identified - by empirical magnitudes, to speak briefly — can meet all the conditions a domain of magnitudes should meet in order for it to be possible to take something akin to a real numbers (that is, something that could be appropriately rendered by a real number) as a univocal measure of it, and this simply because these items could never be enough, and their domains could never display the necessary structural stability, in order to make it possible. ${ }^{26}$

The same difficulties would not arise for non empirical means of measurement of empirical magnitudes. One could, for example, take the distance among two points on a plot of land to measure $\sqrt{2} \mathrm{~km}$, on ground that it counts as the diagonal of a square taken on this same plot of land of side $1 \mathrm{~km}$. But in this way one would actually not measure a physical distance, but the length of a geometrical segment ${ }^{27}$ associated with it on grounds of considerations which fall under the same considerations made above for empirical measures. We would not actually measure a magnitude identified through an empirical enquiry, but a geometrical magnitude associated with an empirical one by appealing to empirical considerations.

But what about geometrical or mechanical magnitudes taken as such? It seems clear that measuring them through some sort of numbers, namely associating a(n exact) measure to them expressed by a numerical term, necessarily requires appealing to the theory of real numbers itself. Geometry and rational mechanics could certainly take on them, independently of any consideration coming from real analysis itself, the task of identifying what is to be measured, but not that of fixing what is to count as a measurement of it, if this is required to be a nongeometrical or mechanical item, in turn.

26 Cf. on this matter, the discussion raised by Hale (2000), among V. Batitsky and Hale himself, contained in Batitsky (2002) and Hale (2002), on which we will come back later, in particular Batitsky (2002), §5, and Hale (2002), §4.

27 Notice that Frege would not have taken a segment to be itself a magnitude. Only its length would have been one. This is perfectly in line with his taking magnitudes to be value-ranges of permutations forming an algebraic structure akin to a group, but conflicts with at least some usual understanding of the term 'magnitude' in geometry and its history. To consider only the most obvious example, in books V and VI of Euclid's Elements, segments (as well as the other geometrical items studied in the first four books) are themselves taken to be magnitudes ( $\mu \varepsilon \gamma \varepsilon$ ₹ $\eta$ ), and this is also so in the whole long history of the theory of proportion up to the early modern age. The conflict depends on the introduction of length (as a separate item) as a third element between the segment and the real number: in absence of length (as a separate item), a real number is directly the measure of a segment, while in its presence, a real number is rather a measure of the segment's length; in the former case, there is no bijection between what is measured (segments) and what measures (real numbers, or, possibly, positive ones if non-positive segments are discarded), in the latter there is a bijection instead, since a real number only measures a single length, though this might be, of course, the length of several segments. As relevant as this difference might be taken to be, none of the points we are raising here essentially depends on it. 
One could finally argue that a suitable account of measurement, in particular of empirical measurement, by something akin to real numbers, does not require that the relevant magnitudes exemplify a domain of magnitudes as rich as (or isomorphic to) the domain of real numbers themselves. All that is required is that some empirical objects exemplify, together with a certain attribute of them (of which they can have different amounts of), a much weaker structure, namely a "measurement structure", whose general characterization and possible refinements are studied in measurement theory (as expounded in Krantz, Luce, Suppes, \& Tversky, 1971-1990, for example). The basic idea here (as described in Batitsky (2002, p. 290 ) and Díez (1997, p. 238)) is that measurement by real numbers is possible if the structure $\left\langle\mathbf{A}, \preceq_{\mathbf{A}}, \oplus_{\mathbf{A}}\right\rangle$-formed by the domain $\mathbf{A}$ of these objects, the binary relation $\preceq_{\mathbf{A}}$ and the binary operation $\oplus_{\mathbf{A}}$ over them, to be interpreted, respectively, as the relation of having less or equal amount of this attribute than, and as a composition, combination or concatenation operation - can be embedded into the structure $\langle\mathbb{R}, \leq,+\rangle$ by a structure-preserving mapping (or homomorphism) $f: \mathbf{A} \rightarrow \mathbb{R}$. The existence of this function is stated by a "representation theorem", a proof of which would, then, be all that is needed to warrant that the objects in $\mathbf{A}$, or, better, the amounts of their relevant attribute, can be measured by real numbers. So conceived, measurement by something akin to real numbers essentially depends on these very numbers, however, since it is nothing but a sort of mirroring into them of an empirical system exemplifying the relevant structure. In a sense, this is an eliminative conception, since it accounts for measurement without appealing to magnitudes conceived of as intermediary entities, as it were, among appropriate physical objects and their measures. At most, one can take these very objects to be magnitudes (though without necessarily exemplifying such a rich structure as that of domains of magnitudes as defined by Frege). ${ }^{28}$

All these considerations seem to suggest that, even if it were admitted that magnitudes (in one sense or another, but in any case conceived as that which is measured) could be precisely identified as such, it would remain that no actual measurement of them (apt to univocally associate a single measure to a magnitude) would be possible, which would not essentially involve real analysis itself. Should we conclude, then, our inquiry, by recognising that applications of real analysis pertaining to the measurement of magnitudes cannot be taken as external?

To further support this conclusion, one could also observe that real numbers outnumber any collection of terms that could be used to communicate the result of a measurement. Of course, we have terms available for this purpose, generally belonging to the vocabulary of real analysis, but no system of terms can be fixed apt to denote the result of whatever measurement of a magnitude, if this measurement were to be considered as measurement by something akin to real numbers.

There are, however, other available understandings of the very idea of an external application of real analysis pertaining to the measurement of magnitudes. In order to see this, it is enough to look at how the terms most commonly used today to denote a real number have initially been employed. These terms have been, indeed, largely used in mathematics

28 One should more properly call these objects 'quantities' then. This situation parallels that described in footnote (27) for geometry, though the relevant geometrical structures are isomorphic with $\mathbb{R}$ or with a closed interval in it, provided, of course, either that their elements are taken to be appropriate equivalence classes of quantities, rather than quantiles themselves, or that, in the definition of these structures, the identity is replaced by the relevant equivalence relation (so as to admit, for example, that a group includes several neutral elements, all equivalent to each other, or several inverses of each of its elements, also all equivalent to each other). 
before disposing of any theory of real numbers in their totality (and even without having any clear idea of what such a totality might look like). These terms have also been, in many different ages of the history of mathematics, pervasively used to denote measures of some magnitudes, typically geometrical ones, or, even, these very magnitudes ${ }^{29}$ conceived as

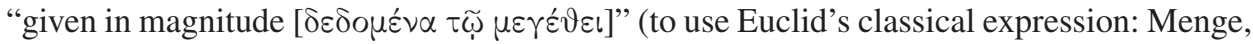
1846, Definition 1; Taisbak, 2003, p. 17). Still, no such use can be taken to presuppose that those terms denote real numbers understood as we do today, that is, as the element of a totally ordered and complete field. One might, then, argue that this pervasive practice in the history of mathematics, that continued up to the very moment in which different definitions of (the field of) real numbers were given, in the second half of 19th-century, prefigured a sort of external application of real analysis, though, as it were, a partial one.

If this were admitted, SFC(Real $\mathrm{mm}_{\text {m }}$ ) should not only be considered as well-stated, but should also be taken as an instance of FC which is at work in Frege's envisaged definition of real numbers.

There is a precise reason for speaking of 'an instance', rather than 'the instance', however. It is the following. Though this way of conceiving an external application of real analysis is certainly plausible, its intrinsic historical character-namely the fact that it appeals to an obsolete mathematical practice, today considered as essentially unjustified, or poorly justified, rather than to a non-mathematical current practice-makes the semantic nature of SFC $\left(\right.$ Real $\left._{\mathrm{mm}}\right)$ quite doubtful, if it is related to such an external application. One could certainly require that an appropriate definition of real numbers has such an obsolete mathematical practice built into itself, in so far as such definition is able to justify post festum that practice. Still, in this case, the requirement would so much be that of ascribing to the relevant terms a meaning apt to recover the one they have within this practice, but rather that of making the definition identify real numbers with the items these terms should denote if such practice has to be directly justified by the definition itself. In other terms, more than a semantic requirement, this becomes a requirement about the relations a theory of real numbers should bear with another theory (or other theories) recovering, in turn, the treatment of the magnitudes (essentially geometrical ones) involved in this practice. But, then, what becomes relevant is not SFC(Real $\left.\left.\right|_{m m}\right)$, but rather another more appropriate instance of FC, which SFC(Real $\mathrm{mm}_{\text {m }}$ ) can only mimic if related to the mentioned historical understanding of the idea of an external applications of real analysis.

We shall come back in $\S 4.3$ to such a more appropriate instance of FC, which Frege's envisaged definition of real numbers seems to comply with. Before this, it is useful to further clarify the foregoing considerations about the possibility of identifying external applications of real analysis pertaining to the measurement of magnitudes, by comparing them with other similar considerations made by other scholars on a similar matter. This is the purpose of the next $\S 4.1 .3$ and $\S 4.2$.

4.1.3. Wright's rejection of Frege's Constraint for real numbers. In \$3.4, we have quoted a passage from Wright (2000) suggesting that Rationale V is not appropriate for definitions of real numbers. It is in order, here, to come back to the argumentative context of this passage.

Just after having stated AC, ${ }^{30}$ Wright claims (Wright, 2000, p. 325):

29 Cf. footnote (27), below.

30 Cf. the quote at p. 102. 
What is it to observe Frege's Constraint? ${ }^{31}$ To insist that the general principle governing the application of a type of number be built into their characterization from the start is in effect just to insist such numbers be characterized by reference to a principle which explains what kind of entities they apply to-are of - and what it is for such entities to be associated with the same or different such numbers. And of course that is exactly what a suitable abstraction principle will do.

When compared with Wright's own quite broad statement of AC, this remark can appear quite strange, since it rather assigns a thoroughly specific nature to this requirement. In our terminology, we might say that, after having stated AC, in its most general form, Wright speaks of it as if he were speaking of FC. This becomes even clearer when he observes that the requirement involves "the thought that the objects of, for example, the classical theories of the natural and real numbers, or of classical geometry, have an essence which transcends whatever is shared by the respective types of models of even categorical (second-order) formulations of those theories" (ibidem, p. 325). This is just what a structuralist view denies. Hence, Wright continues (ibidem, p. 326):

whether the abstractionist should respect Frege's Constraint in recovering a given region of mathematics depends on whether we should think of that region structurally or not.

But when should we not do so? Wright's answer it that we should not when "there is a kind of a priori [...] knowledge which flows from an antecedent understanding of the way [...] [the relevant] concepts are applied" (ibidem, p. 327). Wright further explains this as follows:

It is not that pure knowledge comes first, as the apprehension of an $a$ priori truth about structures, with the applicability of the knowledge so acquired only dawning on one after one has grasped how certain empirical situations can be viewed as, in effect, modeling aspects of that structure. Rather, the content of the a priori knowledge in question already configures concepts drawn directly from the applications [...][so that knowledge] is induced precisely by reflection upon sample, or schematic, applications.

According to Wright, this is just what happens with arithmetic, but not with real analysis (ibidem, pp. 328-329):

[...] it is simply not the case that the distinctive concepts of real analysis can be grounded in their applications after the fashion in which, at least in principle, arithmetical concepts and simple geometrical concepts can. For instance, [...] no real number can ever be given as the measure of any particular empirically given quantity. ${ }^{32}$ There is simply no such thing as determining a real value of a quantity by measurement or indeed by any other empirical procedure - any set of measurements we take will be finite, and even in the best case there will be no empirical distinction between their convergence upon a particular real value as opposed to

31 Cf. footnote (17), above.

32 Wright is here conforming with Hale's use of the term 'quantity' to denote what we rather denote by 'magnitude': cf. footnote (3), above. 
uncountably many others sufficiently close to but distinct from it. [...] the fact is that both our concepts of the identity of particular real numbers and, more importantly, the entire overarching conception of continuity, as classically conceived - the density and completeness of the range of possible values within a parameter determined by measurement-are simply not manifest in empirical applications at all. Rather, so one would think, the flow of concept-formation goes in the other direction: the classical mathematics of continuity is made to inform a nonempirical reconceptualization of the parameters of potential variation in the empirical domains to which it is applied.

This does not only make any instance of FC appropriate for real analysis unable to comply with Rationale V, in its restrictive reading. What is much more important here, is that this also makes SFC(Real $\left.\mathrm{mm}_{\text {m }}\right)$ ill-stated, if the relevant applications are intended to concern empirical magnitudes. Regarding this particular case-which Wright seems to consider as the only relevant one, indeed-, the point he makes seems to be essentially the same we have made above in questioning the possibility that applications of real analysis pertaining to the measurement of magnitudes cannot be external.

Wright goes, however, much farther than this. First, he presents his argument about measurement of magnitudes by real numbers only as an example, but seems then to address his remarks to any (sort of) application of real analysis. Second, he openly dismisses AC (and, then, FC) as an advisable guide for a suitable definition or real numbers. Both points exceed our present proposal. The first entails that no application of real analysis can be external, which is not something we argue for. The second implies that either Frege was wrong in endorsing FC in relation to his envisaged definition of these numbers, or possibly that he did not do it, in fact, whereas we would tend to exclude both possibilities. On the one side, it seems, indeed, unquestionable to us that Frege endorsed FC regarding real numbers. On the other side, although Frege could certainly have been wrong in this respect (as in others), he needs not be as wrong as Wright's remarks suggest, since none of Wright's arguments seem to exclude the possibility that either SFC $\left(\operatorname{Real}_{\mathrm{mm}}\right)$ be conceived as suggested at the end of $\$ 4.1 .2$, above, and that, when so conceived, it results in an admissible requirement, or that some other instance of FC could provide an appropriate guide for a definition of real numbers. ${ }^{33}$

4.2. Real numbers and empirical magnitudes: The Batinsky-Hale debate. On the last point, Wright openly differs from his frequent coauthor Hale, who holds that (Hale, 2002, p. 307)

Frege was 'substantially right' 34 in his belief that the application of reals as measures of quantities is essential to their very nature, and so should be built into an adequate definition of them. ${ }^{35}$

33 Critical discussions (beyond those to be recalled in the next section) of Wright's argument, both as regards the ensuing interpretation of Frege's views and the role of FC, and as regards the additional claim, argued for by Wright in the same article, that ability of meeting FC can discriminate between neo-logicist and (ante rem) structuralist characterizations of natural and real numbers, can be found in Hale (2016), Snyder et al. (2018), Sereni (Manuscript).

34 The quotations marks signal a quotation from Hale (2000, p. 104).

35 It is a further question whether Hale and Wright understand FC in the same way. 
This claim is made in an article which appeared two years later than that quoted at the beginning of $\S 1$.

The main proposal of the older article is to advance a (consistent) definition of real numbers which complies with FC and follows Frege's indications by being based on a preliminary definition of domains of magnitudes and by identifying real numbers with ratios on such domains, though being different from Frege's in many relevant aspects. One difference concerns the way domains of magnitudes are fixed. It is possibly to emphasize this difference that Hale labels them, when defined in his own way, 'complete quantitative domains', and define them by stages by identifying a number of stricter and stricter conditions characterizing different structures for different sorts of "quantitative domains" (Hale, 2000, pp. 106-108). Though this terminology is not Frege's, it will be convenient to adopt it here, for the sake of clarity. From now on, we shall call, then, 'complete domains of magnitudes' those domains allowing to define real numbers as ratios on them (up to now merely called, after Frege, 'domains of magnitudes'), in order to single them out among a larger variety of domains of magnitudes.

The new article is a reply to Batitsky (2002), who had criticized Hale's definition of real numbers by retorting that his endorsement of FC leads him to two untenable commitments: "that there can be no satisfactory explanation of measurement application of real numbers to basic ontologies from which reals cannot be constructed by suitable logicist abstractions"-for example finite ontologies or infinite ones which display a structure weaker than that of complete domain of magnitudes - and and that the "existence of at least one" such domain is "necessary and a priori", since so is "the existence of reals (at least for logicists)" (ibidem, p. 289).

Batitsky is of course right in judging the former commitment untenable, but he is also wrong in arguing that endorsing FC leads to it: requiring that a definition of real numbers have built into it the applications of real analysis pertaining to the measurement of magnitudes is of course not the same as denying that these applications might be accounted for in alternative ways, for example, as Batisky maintains, through representation theorems, in line with measurement theory (cf. pp. 122-123, above).

As regards the latter commitment, things are subtler. Batitsky takes it to be manifestly untenable since he takes for granted (in agreement with Wright, apparently) that magnitudes cannot but be empirical items, which is completely alien both to Frege's and Hale's views: so much alien that both believe it is possible to prove that a complete domain of magnitudes necessarily exists by showing how to construct it (a priori) based on natural numbers (Frege, 1903, §II.164; Hale, 2000, §3). Moreover, such a commitment follows from FC only in the presence of supplementary metaphysical assumptions, which Frege certainly endorsed but which are, as such, independent of it (at least if it is taken to be motivated by Rationale IV in its liberal reading).

What is more relevant for our proposal is, however, not this nor, more generally, the way Hale (convincingly) responds to Batitsky's allegations. It is rather that these allegations force Hale to acknowledge that in his older article he had "made no attempt to elucidate or defend Frege's Constraint" (Hale, 2002, p. 307), and to try to fill the gap. Here is where he starts from (Hale, 2002, pp. 309-310):

There is, of course, a more-or-less obvious price to be paid for so characterizing quantitative domains that it is not only impossible to show that physical quantities of any kind - such as lengths, masses, etc.constitute such a domain [...], but virtually certain that they do not do so. For it is an immediate and unavoidable consequence of this negative 
point that any explanation of the applications of reals (defined as ratios of elements of a quantitative domain) in the measurement of physical quantities must be considerably less straightforward than the explanation that is rendered possible by the introduction of cardinal numbers by means of Hume's principle of their empirical applications.

This remark points in the same direction as Wright's: it emphasizes that there is a striking asymmetry between the way arithmetic and real analysis, respectively, relate to their canonical applications, at least if those of the latter pertain to measurement of empirical magnitudes. Still, whereas Wright takes this asymmetry as a sufficient ground for accepting FC for natural numbers but not for real ones, Hale considers it as a reason for looking for an elaborate enough way for explaining measurement of empirical magnitudes by reals based on the definition of them as ratios of magnitudes.

He begins with distinguishing "three possible readings" of FC (Hale, 2002, p. 312):

There is, first, an entirely anodyne reading on which the Constraint demands no more than, and would be met in full by, the availability of representation theorems of the kind proved in standard measurement theory. Second, there is the exorbitant reading which requires, if the reals are to be defined by abstraction, that the abstraction should be over an equivalence relation or relations holding among suitably chosen actual lengths, masses, etc. Third, there is a reading on which the Constraint requires more than the first but appreciably less than the second: that an adequate definition of the reals must, as Dummett puts it, ${ }^{36}$ "display the general principle underlying the use of the real numbers to characterise the magnitude of quantities of these and other kinds" [...].

It is of course the third reading that interests us. Hale motivates as follows his claim that FC, on this reading, is met by his own definition of real numbers while still considering this definition as apt to explain the application of real analysis to the measurement to empirical magnitudes (Hale, 2002, pp. 313-314):

$[\ldots]$ any explanation of the use of real numbers in measuring [...][physical quantities $\left.{ }^{37}\right]$ must, if the reals are defined as ratios of elements of a domain, be more or less indirect. [...] How, then, are empirical applications of the reals to such quantities to be explained? In a nutshell, [...] quite simply: by observing that whilst physical quantities of any given kind do not—and arguably could not—exhibit the abstract structure of even a minimal, much less a, quantitative domain, this does not mean that they cannot constitute a partial realisation of it.

Hale illustrates what he means by 'partial realization' by considering the example of masses. In short, he takes a kind of empirical magnitude, e.g., mass, to be defined through an abstraction principle of the form

$$
\text { ' } \forall x, y\left[\phi_{A}(x)=\phi_{A}(y) \Leftrightarrow x \text { is just as } A \text { as } y\right] ',
$$

where ' $x$ ' and ' $y$ ' vary over appropriate empirical objects, $A$ is an appropriate attribute of these objects, and $\phi_{A}$ is the kind of magnitude to be defined; and seems to argues that,

36 Cf. the passage from Dummett (1991) quoted in $\S 1$.

37 Cf. footnote (3), above. 
whatever kind of magnitudes $\phi_{A}$ might be, these objects and attribute are (because of the very notion of a kind of magnitudes) such that a structure $\left\langle\phi_{A}, \preceq_{\phi_{A}}, \oplus_{\phi_{A}}\right\rangle$ can be defined, which is homomorphic with a complete domain of magnitudes. ${ }^{38}$ According to him, this is enough to explain the applicability of real numbers to the measurement of $\phi_{A}$ 's.

It is not important here to assesses whether this account is appropriate, in general (we leave this to be decided by experts in the measurement). What is important is the basic idea it seems to convey, which seems to conform with Frege's views (which certainly did not depend on a more accurate analysis of the general notion of a magnitude: Dummett, 1991, pp. 278-291): according to FC, real numbers are to be defined as ratios over complete domains of magnitudes; provided that it is "virtually certain" that no empirical magnitudes can "constitute" such a domain, this means that real numbers are to be defined as ratios of nonempirical magnitudes; on the one side, this is enough to directly explain their application to the measurement of (non-empirical) magnitudes forming such a domain; on the other side, this provides a basis for indirectly explaining their application to the measurement of empirical magnitudes, by showing that they form a structure homomorphic to a complete domain of magnitudes. ${ }^{39}$

But, if this is so, the crucial point in the explanation of the applications of real analysis pertaining to measurement of magnitudes rests, also for Hale, on the proof of appropriate representation theorems establishing an homomorphism among two structures, one of which - that of a complete domain of magnitudes - is fixed and prescribed by the very structure that real numbers are expected to form, namely by the requirement that it be appropriate for allowing for a definition of these numbers as ratios on it. This makes the difference between Hale's neo-Fregean account and that of the partisans of the measurement theory quite thin: where the latter take the relevant representation theorems to directly connect structures defined on the basis of empirical objects with real numbers, the former takes these theorems to connect these structures-as well as other ones involving nonempirical objects or defined on the basis of them-with the structure of a complete domain of magnitudes, which is, in turn, connected to real numbers by the definition of these numbers as ratios on such a domain. In the latter account, then, real numbers directly enter the game only in connection to the single structure of complete domains of magnitudes, and it is only with respect to their applications to the measurement of (non-empirical) magnitudes complying with this structure that their definition is to be judged. Since, if the nature this definition ascribes to these numbers makes it possible, in itself, to (directly) justify

38 Of course, if we take here (as it seems we should do) the relevant magnitudes to be the different values that the function $\phi_{A}$ can take, rather than the empirical objects themselves on which this function is defined, then these magnitudes are abstract objects. Calling them 'empirical' could, then, be questioned. Still, one can take this to be licensed by the fact that our capacity of establishing whether an (empirical) object $a$ is just as $A$ as another (empirical) object $b$, and, then, whether $\phi_{A}(a)$ is the same magnitude as $\phi_{A}(b)$, depends on our implementing empirical procedures.

39 No clear effort is done here to elucidate the notion of a magnitude, in general. Hale seems to take it for granted both that nonempirical magnitudes are just the elements of a domain of magnitudes (which would make the very notion of nonempirical magnitudes not forming such a domain nonsensical), and that empirical ones are always elements of a structure $\left\langle\phi_{A},+_{\phi_{A}},<_{\phi_{A}}\right\rangle$ homomorphic to such a domain, by leaving to empirical enquiries the task of identifying both the (empirical) objects over which empirical magnitudes of a certain kind are defined by abstraction, and the relevant attribute of them, as well as that of explaining how a statement like ' $a$ is just as $A$ as $b^{\prime}$ ' (where $a$ and $b$ are whatever objects having the attribute $A$ ) is to be understood in any particular case, and that of fixing the truth-conditions of such a statement. 
their application to measurement of these (non-empirical magnitudes), it will also make possible to (indirectly) justify their application to measurement of empirical magnitudes, because of the homomorphism holding between the relevant structure involving these latter magnitudes and that of complete domains of magnitudes. ${ }^{40}$

This suggests taking FC to require, in the case of real numbers, that their definition be such to make these numbers intimately connected to a (non-empirical) theory of complete domains of magnitudes, since, according to such an account, this theory could be taken as a general theory of (any sort of empirical and non-empirical) magnitudes, at least if the aim of such a theory were taken to be that of fixing the general notion of a magnitude in relation to its being a possible object of measurement. If such requirement is met, real analysis would be connected not only to this very general theory, but also, via its intermediary, to geometry and rational mechanics (for the magnitudes they deal with form complete domains of magnitudes), and to any empirical theory presiding over the measurement of a certain kind of empirical magnitudes.

4.3. Architectonic Frege's Constraint. More generally, this suggests an alternative specification of FC as complying with Rationale VI in so far as this prescribes an appropriate architectonic of (a portion of) mathematics, according to which the canonical applications of a certain theory are explained by the way this theory is connected to another because of the definition of the very objets it deals with.

According to such a specification, FC would appear as the requirement that the relevant definitions be such that the canonical applications of the relevant theory be built into the very nature they ascribe to the objects they define in so far as they make these objects result from ingredients involved in an antecedent (and, then, more basic) theory providing the elements for a general characterization of the subject-matter of these applications.

Call 'Architectonic FC' or 'AFC' for short, the general pattern that the instances of FC depending of this specification obey to.

4.3.1. Architectonic Frege's Constraint for real numbers. If we take the canonical applications of real analysis to be those pertaining to measurement of magnitudes, the appropriate instance of AFC for real numbers would be the following:

[AFC $\left(\right.$ Real $\left.\left._{\mathrm{mm}}\right)\right]$ Suitable definitions of real numbers, as objects real analysis is about, must be such to ascribe an intrinsic nature to (each of $)^{41}$ these numbers, and to do it in such a way that the applications of real analysis pertaining to the measurement of magnitudes be built into this very nature in so far as they make these objects specified based on a general theory of magnitudes, so that they work as measures of whatever sort of magnitudes.

Needless to say: Frege's envisaged definition of real numbers perfectly complies with this constraint. One could even consider that it does that too perfectly, as it were, namely, that this constraint is stated ad hoc in order to make this definition comply with it. This would be so, however, only if the foregoing account of the way in which a general theory of magnitudes, providing a characterization of complete domains of magnitudes, enters an explanation of the measurement of any sort of magnitudes by real numbers were blanked out.

40 Cf. footnote (39), above.

41 Cf. footnote (21), above. 
As we have already said, it is not our purpose, here, to support such an account. What is relevant is just that it can be plausibly taken to reflect Frege's views on the matter. ${ }^{42}$

This does not only suggest that Frege actually endorsed AFC $\left(\right.$ Real $\left.\mathrm{mm}_{\mathrm{m}}\right)$, but also that this is just the instance of FC which is at work in Frege's envisaged definition of real numbers. It is moreover easy to see that, once external applications of real analysis are conceived as said at the end of $\S 4.1 .2, A F C\left(\right.$ Real $\left._{\mathrm{mm}}\right)$ agrees with SFC $\left(\right.$ Real $\left._{\mathrm{mm}}\right)$, so that the latter might be seen, as it were, as an alias of the former.

It is, then, fair to say that Frege conceived FC, in relation to a definition of real numbers, as requiring not only that these numbers be defined as ratios on, and then measures of, (non-empirical) magnitudes, but, just because of this, he also maintained:

i) that these numbers be, by definition, both distinct from the magnitudes they measure (though he also envisaged to define them so as to make they form a complete domain of magnitudes: Frege (1903, §II.245)), ${ }^{43}$ and independent of natural numbers (though he also envisaged, as observed above (p. 127), to prove that appropriate complete domains of magnitudes necessarily exist by constructing one of them based on natural numbers);

ii) that real analysis have a quite precise place in the architectonics of mathematics, namely that it be connected from above with a general theory of magnitudes, thus being rather essentially independent of arithmetic, though sharing with it a subjacent system of logic.

42 After having lamented that "what a magnitude is surely has never been stated satisfactorily" (Frege, 1903, §II.160; Frege, 2013, p. 1572, §II.160), having observed that this depends on having asked "the wrong question", and having suggested that the good one is "how must a concept be constituted in order for its extension to be a domain of magnitudes" (Frege, 1903, §II.161; Frege, 2013 , p. $158_{2}$; cf. Hale's passage quoted at p. 98, above), Frege abruptly cuts down the discussion by admitting that "since our initial concern is to obtain a foundation just for the theory of the real numbers, we will simplify the matter by leaving absolute magnitudes out of consideration, focusing only on those domains of magnitudes in which there is an opposition corresponding to that between the positives and negatives for the measuring numbers" (Frege, 1903, §II.162; Frege, 2013 , p. $159_{2}$ ). This suggests that Frege was thinking that a definition of complete domains of magnitudes acquiescing with this abrupt simplification would have provided a general framework to which other cases could be reduced (on this matter, cf. Dummett (1991, pp. 278-281)), which is in line with Hale's account. An important proviso has to be made, however. Both Frege's and Hale's definitions of domains of magnitudes are structural in the sense that they merely fix the structural conditions that such domains are to meet as a whole, without also fixing the ultimate nature of the elements of such a domain. Still, while, for Hale, such elements are unspecified objects meeting these structural conditions, when taken all together, Frege requires, as said in $\S 4.1 .2$, that these elements be extensions of permutations, and suggests that in a consistent faithful rephrasing of his definitions these elements will be permutations (cf. footnote (22), above). Frege's original definition advances no requirement about the specific nature of the objects which these permutations are supposed to act on, and so it will be for a consistent rephrasing of it: this is just what makes these definitions structural. Still, one should not forget that, in proposing this requirement, Frege advances a condition that is absent from Hale's definition. For this condition to be met, Hale's characterizations of a kind $\phi_{A}$ of empirical magnitudes and of the corresponding structure should be appropriately amended. It is not important, here, to consider how this could be done. What is important is to observe that this would have no consequences on the aspects of the foregoing account which are relevant for our present purpose.

43 According to Frege's original definition, this would require defining also real numbers as extensions of permutations, which can only be done thanks to a trick making their definition far from natural: cf. Dummett (1991), p. 291. 
iii) that a pervasive practice in history of mathematics, up to the second half of the 19th century, consisting in using numerical terms to denote measures of geometric magnitudes, or these very magnitudes, be recovered and justified post festum.

§5. Rethinking Frege's foundational program. After having argued that $A C$ played an essential role in Frege's foundational endeavor in the form of appropriate instances of FC, in $\S 4.1$, we have wondered which instances of this last constraint could have been at work as guides for his definitions of natural and real numbers, respectively. Our enquiry has led us to conclude that whereas the instance $F C$ guiding the former definition is an instance of SFC, namely SFC( $\left.\mathrm{Nat}_{\mathrm{tc}}\right)$, no instance of SFC could have guided the latter definition, if not under an understating that makes it mimic a non-semantic requirement. This definition should have rather been guided by an instance of AFC, namely AFC $\left(\operatorname{Real}_{\mathrm{mm}}\right)^{44}$ —which is just the requirement mimicked by the relevant instance of SFC for real numbers. It is now time to ask whether this difference reflects itself on how Frege's foundational endeavor is depicted in the two cases.

This endeavor is generally seen as governed by a unique foundational program, labeled 'logicism', and often depicted as a primarily philosophical enterprise, essentially concerned with accounting for the nature of mathematical objects via semantic considerations, aimed at justifying our epistemic access to such objects.

Both this label and this picture might be questioned, and this both concerning Frege's definitions of natural and real numbers. ${ }^{45}$ Here we would like, however, to focus only on their appropriateness in relation to the latter definition, and on the way a faithful understanding of the motivations guiding this latter definition might reverberate on our understanding of the motivation guiding the former.

At the end of $\$ 4.1 .1$, we have already observed that there is room for taking Frege's proposal of getting arithmetic within a system of logic as prompted by SFC $\left(\mathrm{Nat}_{\mathrm{tc}}\right)$, rather than the other way around, which makes this last constraint comply with Rationale VI. But what about Frege's envisaged definition of real numbers and the instance of FC that should have guided it, namely AFC $\left(\right.$ Real $\left._{\mathrm{mm}}\right)$ ?

In so far as Frege's definition of domains of magnitudes is entirely achieved within his system of logic (at least in so far Basic Law V is taken to be a law of logic, and to be, then, part of this system), AFC(Real $\mathrm{mm}_{\text {m }}$ ) certainly prescribes to connect real analysis to logic. This is possibly enough for maintaining that the label 'logicism' fits with his definition of real numbers. But it is much more doubtful that $\mathrm{AFC}\left(\mathrm{Real}_{\mathrm{mm}}\right)$ could be taken, also in

44 That Frege could not have intended FC just in the same way for natural and real numbers had been already suggested by G. Currie (Currie, 1986, p. 361):

$[\ldots]$ we see an essential difference between the account of the applicability of the natural numbers and that of the real numbers. The explanation of the applicability of a natural number $n$ is local; it depends only on what concepts fall under the concept of which $n$ is an extension. But the explanation of the applicability of a real number $r$ is global; it depends upon the existence of a mapping from the real numbers into the structure which is the particular magnitude kind we are considering.

This characterization of the difference is misleading, but the basic idea is correct, we think. What we have tried to make is to give more substance to it.

45 Concerning the former numbers, this matter is the object of a seperate article of one of us: Panza (2018). A larger article tackling the question for both sorts of numbers is in preparation. 
Frege's perspective, as prescribing a definition of real numbers as logical objects. There are different reasons for that.

Two of them are rather technical.

Here is the first. One could observe that, whereas Frege defines natural numbers in such a way that each one of them is identified with a particular and univocally specified objectnamely, with the extension of the concept of being the extension of a(first-level) concept equinumerous with a certain finite first-level concept: Frege (1903, §I.40-\$I.46) ${ }^{46}$ - he does not define (complete domains of) magnitudes in such a way that this also happens for each (element of such a domain of) magnitude(s), just because his definition is structural in nature. ${ }^{47}$ It follows that, in Frege's perspective, magnitudes cannot be, at the same time, univocally specified and logical objects: if they are taken to be logical objects, they have to be taken to be merely structural objects, places in a structure; if they are taken to be univocally specified, they have to be taken to be elements of a particular system exemplifying, possibly partially, the structure of domains of magnitudes (this should have been already clear from what we have said at the beginning of \$4.1.2). ${ }^{48}$

This has a consequence for the very nature of ratios of magnitudes, that is, for what Frege prescribes real numbers to be. The way he envisaged defining them would have made any

46 The definition suggested in the Grundlagen is slightly different, as it is well-known. This difference does not affect our present point, however.

47 Cf. footnote (42), above.

48 It is interesting to notice that this way of conceiving Frege's purported definition of real numbers - also according to what specified in footnote (42) - echoes some of Frege's objections to Hilbert's treatment of the axioms of Euclidean Geometry. In Frege (1903, pp. 374-375), Frege observes that what Hilbert has really accomplished with his purported axioms, in his Grundlagen der Geometrie, is not to define first-level concepts, e.g., the concept of a point, thus giving means for identifying the nature of specific geometrical objects, but rather to introduce a number of characteristics for such first-level concepts (cf. also Frege's letter to Hilbert of 6.1.1900, in Frege, 1976, pp. 73-74). In Frege's technical sense, "a characteristic of a concept is a property an object must have if it is to fall under that concept" (Frege, 1903, p. 373; Frege, 1971, p. 35). Characteristics can be attributed to concepts of any level, and will select properties of objects, if they are characteristics determined by first-level concepts, and properties of concepts of level $n$ if they are characteristics determined by concepts of level $n+1$. According to Frege, what Hilbert has at most accomplished is to introduce certain characteristics of first-level geometrical concepts, i.e., characteristics that different first-order concepts of, for instance, point must satisfy in order to fall under a given second-level concept, a concept like that of being a concept of point' (Frege does not explicitly mention this, although he of course laments the confusion engendered by Hilbert in using 'point' to denote both concepts: Frege, 1903, p. 374; Frege, 1971, p. 37). Such definition does not also provide characteristics of the first-level concepts of point, i.e., the properties that geometrical objects, i.e., points, must possess in order to fall under such first-level concept.

One way of reading this is to say that by giving definitions of second-level concepts under which first-level geometrical concepts fall, Hilbert has not managed to identify points with specific objects, but merely to say how different concepts of such objects must behave in order to be among concepts of one or other geometry. Arguably, something similar is what has been said above about Frege's definition of domains of magnitudes. To repeat ourselves, Frege's definition of domains of magnitudes is structural in the sense that it merely fixes the structural conditions that such domains are to meet as a whole, but does not also determine the ultimate nature of the elements of such a domain: in other words, it only establishes the properties that concepts of magnitudes must have in order to fall under the concept of domain of magnitudes.

This is not the place to explore the consequences of such an analogy. However, it is remarkable to see Frege criticizing Hilbert for giving a definition of geometrical concepts which so much resembles his own definition of domains of magnitudes. 
ratio on a particular system exemplifying the structure of domains of magnitudes identical with a ratio on another such system. But if the elements of these systems are not logical objects, how might their ratios be so? And, analogously, if magnitudes as logical objects are not univocally specified, how might their ratios be so?

Here is the second. For real numbers to be able to be defined at the same time as ratios of magnitudes and as objects existing a priori, at least a domain of magnitudes is to be warranted to exist a priori. Still Frege's (as well as Hale's) definition(s) of domains of magnitudes cannot warrant, as such, that a non-empty such domain, and, a fortiori a complete one, exists. This makes a definition of real numbers as ratios of magnitudes require an independent existence proof (which is not at all the case for Frege's definition of natural numbers as cardinals of concepts). But, how might objects whose definition requires an independent existence proof be logical objects? ${ }^{49}$

But there are also more general arguments concerned with Frege's conception of logic.

For Frege, logic is characterized by its generality. Still, this can be understood in different ways. According to Goldfarb, logic is general, for Frege, since, for him, its "business [...] is to articulate and demonstrate [...] logical laws" and these are "universal", for "they are applicable to any subject matter", and they are "general, not in being about nothing in particular [...], but in using topic-universal vocabulary to state truths about everything", even "supremely general", for, "aside from variables, all that figure in them [...] figure in discourse on any topic whatsoever" (Goldfarb, 2001, p. 26). According to MacFarlane, instead, "the kind of generality that distinguishes logic [...] is a generality in the applicability of the norms it provides", and comes from its being "normative for thought as such" (MacFarlane, 2002, pp. 37-38), or, as Frege himself says in the foreword of Grundgesetze, from its "prescribing how to think wherever there is thinking in general" (Frege, 1903, Vorwort, p. XV; Frege, 2013, p. XV 1 ).

Now, whichever of these two (in a sense, opposite) accounts one favors, it seems clear that no statement about magnitudes can be taken to be a law of logic, simply because of its lacking the sort of generality which logic requires. ${ }^{50}$

Indeed, Frege's definition of complete domains of magnitudes is certainly achieved within his system of logic (provided Basic Law V be taken to be part of it), but, as observed by Shapiro, this system is used "to recapitulate something sufficiently resembling

49 On this matter, more is said in Boccuni \& Panza (Manuscript).

50 Despite the fact that Frege provides a formal definition of domains of magnitudes entirely within his formal system, and that this definition can be consistently rephrased within a quite weak system of third-order logic (see Boccuni \& Panza (Manuscript)), this is, and could not but be, a definition of the pure structure that domains of magnitudes are supposed to meet, not of these very domains. The characterization of each of the latter cannot, indeed, but depend on nonlogical resources. Moreover, from the mere fact that this structure can be defined within a system of logic it does not follow at all that it is logical in nature. Its definition within this system merely appeals to structural properties of binary relations (their being invertible, composable, and the fact that identity is among them) to mimic the conditions to be met by appropriate groups of permutations. But this is certainly not enough to make the notion of such a group as general as a logical notion should be (at least according to a Fregean conception of logic). At most, one could follow Kant in arguing that "the concept of a magnitude" is "the consciousness of a homogeneous manifold in intuition in general, in so far as through it the representation of an object first becomes possible" (B, 203; Kant, 1998, p. 287), and then conclude that a priori judgements about magnitudes are a necessary condition for sensible intuition. But it seems that one could hardly maintain that these judgments are "applicable to any subject matter" or that they are "normative for thought as such". 
metatheory", for, here, "Frege anticipated a technique now attributed to Ramsey" according to which "one replaces an axiomatization with an explicit definition of a second-level concept, that is, a relation on relations" (Shapiro, 2000, p. 345). To be more precise, Frege defines a complete domain of magnitudes as the extension of a concept so shaped that under it fall extensions of permutations that, when taken all together, meet some appropriate conditions, all expressible in terms of the operator of inversion of a binary relation and of the operation of composition of two such relations. In this way, he transforms algebraic axioms (characterizing an appropriate group) in an explicit definition. This allows him to avoid any existential assumption and so to give to his definition the external appearance of a logical definition. But this does not change the substance of what he does (as showed, by the way, from the fact that an independent existence proof is needed, as observed above): he fixes a particular structure, so departing, in fact, from the prerogatives of logic. Moreover, passing from complete domains of magnitudes, so defined, to ratios on them, namely to real numbers, requires adopting a new appropriate abstraction principle (presumably rephrasing Euclid's definition of proportionality, that Frege shows to be familiar with, since he mentions it in the Grundlagen: Euclid, Elements, Definition V.5; Frege, 1903, $\S 19$; Simons, 1987, p. 40; Dummett, 1991, pp. 290-921), which could hardly be taken, in turn, as a logical law.

All this suggests that, if some form of logicism encompasses both Frege's definition of natural numbers and the envisaged one of real numbers, this is quite different in the two cases. True: both the former definition and a preliminary, though essential, part of the latter are articulated within the same formal system, which Frege considered as a system of logic. Still, while the former defines natural numbers as objects fixed by genuinely explicit definitions (Basic Law V being taken for granted and apt to license the appeal to extensions, this preliminary part of the latter has merely the external form of an explicit definition, and it does define, in any case, no univocally specified object.

This difference can be explained by coming back to rationale VI. We have suggested that Frege's definition of natural numbers could be seen as prompted by SFC(Nat $\mathrm{N}_{\mathrm{tc}}$ ), and that this can, in turn, be taken to comply with this rationale, since it requires that these numbers be defined within a general theory of sortal concepts and objects (cf. the end of $\S 4.1 .1$, above). In so far as this is certainly also the case of AFC $\left(\operatorname{Real}_{\mathrm{mm}}\right)$, which can be taken to prompt Frege's envisaged definition of real numbers, this results in acknowledging that the two definitions have this in common: both are intended to meet a requirement that is (partially, at least) motivated by this rationale, i.e., both aim to realize an appropriate architectonics of mathematics, both in itself, and within knowledge as a whole. On this respect the essential difference is this: while the definition of natural numbers is aimed at including their theory within logic, conceived as a general theory of sortal concepts and objects, that of real numbers merely uses logic as a convenient tool to articulate the relations of the theory of these numbers with a general theory of magnitudes, or theory of complete domains of magnitudes, on the one side, and with geometry, rational mechanics and empirical enquiries, on the other.

This is confirmed by a counterfactual remark, as it were. Had Frege aimed to include also real analysis within logic, he would have plausibly chosen a much simpler road: that of defining these numbers as arithmetical objects, for example as pairs composed by a natural number and a set of natural numbers. ${ }^{51}$ In sketching the existence proof that a non-empty complete domain of magnitudes exists (Frege, 1903, §II.164), he envisaged,

51 For such a definition, cf. von Kutschera (1966), and, for a variant of it, Panza (2016), pp. 415-419. 
indeed, to have recourse to such pairs, ${ }^{52}$ which suggests he considered them, if not as logical objects, at least as objects whose existence is warranted by logic, or, in any case a priori. This would have made, however, real analysis result from arithmetic, rather than from a general theory of magnitudes, or theory of complete domains of magnitudes, so contravening to SFC(Real $\left.\mathrm{mm}_{\mathrm{m}}\right)$.

Would this also have contravened any other possible instance of FC? Possibly not.

After having briefly indicated the road he would have followed to define complete domains of magnitudes (Frege, 1903, §II.162), Frege illustrates his idea by the example of distances on a straight line, or rectilinear length (ibidem, §II.162). This is, of course, a quite specific, and very simple case, which is of no help in getting a general notion of a magnitude (Dummett, 1991, p. 280), but the fact that Frege considered it suggests that he took it as representative enough.

Let, then, $\mathbf{A}$ be a domain of objects having an attribute $A$ from which a kind of magnitude $\phi_{A}$ can be somehow extracted, conforming, for its structural properties, to this example (one could take the elements of $\mathbf{A}$ to be segments of straight lines, polygons, periodic intervals of time, or mass bodies, and $\phi_{A}$ rectilinear length, area, time duration and mass, respectively). Let also ' $\prec_{A}$ ' and ' $\oplus_{A}$ ' denote, respectively, an appropriate strict-order relation and an appropriate operation of composition or concatenation on $\mathbf{A}$ (from the former of which external multiplication can be defined by $k x=\underbrace{x \oplus_{A} x \oplus_{A} \ldots \oplus_{A} x}_{k \text { times }}$ and $\frac{1}{k} x=y \Leftrightarrow k y=x$, were $x$ and $y$ are any element of $\mathbf{A}$ and $k$ any natural number). A quite natural way to measure an element $a$ of $\mathbf{A}$ is by ascribing to it a value of $\phi_{A}$ in the form of an infinite sum of rational numbers, proceeding this way:

- Choose another element $u$ of $\mathbf{A}$, to be used as a unity of measure, and look for a natural number $n$ such that $n a \prec_{A} a \preceq_{A}(n+1) u$;

- Look for an element $r_{1}$ of $\mathbf{A}$ such that $a=n u \oplus_{A} r_{1}$, and put

$$
\phi_{A}(a)_{[u]}=n+\phi_{A}\left(r_{1}\right)_{[u]} ;
$$

(by then admitting that, for any two elements $x, y$ of $\mathbf{A}, \phi_{A}\left(x \oplus_{A} y\right)_{[u]}=$ $\left.\phi_{A}(x)_{[u]}+\phi_{A}(y)_{[u]}\right)$;

- Evaluate whether $r_{1} \preceq_{A} \frac{1}{2} u$ or $\frac{1}{2} u \prec_{A} r_{1} \preceq_{A} u$, look for an element $r_{2}$ of A such that $a=n u \oplus_{A} r_{2}$, in the former case, or $a=n u \oplus_{A} \frac{1}{2} u \oplus_{A} r_{2}$, in the latter case, and put, respectively

$$
\phi_{A}(a)_{[u]}=n+0+\phi_{A}\left(r_{2}\right)_{[u]} \quad \text { or } \quad \phi_{A}(a)_{[u]}=n+\frac{1}{2}+\phi_{A}\left(r_{2}\right)_{[u]} ;
$$

- Continue indefinitely this way, so as to get the equality:

$$
\phi_{A}(a)_{[u]}=\sum_{i=0}^{\infty} \lambda_{i} \frac{1}{2^{i}}
$$

with

$$
\lambda_{0}=n \quad ; \quad \lambda_{i}=\left\{\begin{array}{cc}
0 \quad \text { if } \quad r_{i} \preceq_{A} \frac{1}{2^{i}} u \\
1 \quad \text { if } \quad \frac{1}{2^{i}} u \prec_{A} r_{i} \preceq_{A} \frac{1}{2^{i-1}} u
\end{array} \quad(i=1,2, \ldots) .\right.
$$

52 What is called 'set' here is called 'class [Klasse]' by Frege and identified with the extension of a concept. 
Let, now, $\Lambda$ be the set of (positive) natural numbers $\left\{i: \lambda_{i}=1\right\}$ (or, in Frege's terminology, the extension of the concept of being a positive natural number $i$ such that $\left.\lambda_{i}=1\right){ }^{53}$ It is easy to see that the (Cauchy) series of the form $\sum_{i=0}^{\infty} \lambda_{i} \frac{1}{2^{i}}$, providing measures for the elements of $\mathbf{A}$ (relative to $u$ ), are in bijection with the pairs like $\langle n, \Lambda\rangle$. These pairs are just those involved in Frege's envisaged existence proof. Hence, defining real numbers as such pairs would have permitted to explain a priori the application of these numbers to the measurement of magnitudes behaving as rectilinear length (which Frege himself seems to take as paradigmatic), and, thus, to meet a plausible instance of FC for these numbers, concerned with their application to measurement of magnitudes.

Still, as plausible as such an instance of FC might be, it would not have prescribed a definition connecting real analysis with a general theory of magnitudes, or theory of complete domains of magnitudes, and, through it, to geometry, rational mechanics and empirical theories: the explanation of its applications pertaining to measurement of magnitudes which it would have allowed would have only involved an informal account of the way measures of magnitudes could be expressed by Cauchy series of rational numbers, in important but still particular cases.

And, if we understand well his point (his argumentation, here, is far from crystal-clear), this is just the main objection Frege makes to Cantor's definition of real numbers through Cauchy series of rationals (Frege, 1903, §II.68-§II.85; Dummett, 1991, pp. 263-276).

Frege seems here to reject the very idea that a series of rationals could be taken to be a number, as he would have possibly also rejected that this could be the case for a pair like $\langle n, \Lambda\rangle$ : at most they could be associated with numbers, which would require an independent identification of the (real) numbers to which they would be associated. This line of thought is not entirely convincing, however: why should one be licensed to take natural numbers to be extensions of concepts, and real ones to be ratios of magnitudes, and not to take the latter to be pairs like $\langle n, \Lambda\rangle$ ?

It seems, therefore, that the crux of Frege's rejection of the aforementioned option, as well as of Cantor's definition, cannot be here. His point is rather that, if real numbers were so defined, there would be no general explanation of their being essentially measures of magnitudes. Since a procedure as the foregoing can intelligibly result in associating a Cauchy series of rationals, or a pair like $\langle n, \Lambda\rangle$ to the relevant magnitude of $a$, only in so far as it is already clear what a magnitude of an element of $\mathbf{A}$ is; and this series can intelligibly provide a measure of this magnitudes, only in so far as it is already clear how a series of rationals can provide a measure of a magnitude. But how could both things be made clear without a general theory of magnitudes and a consequent definition of their measures as ratios on them?

Here is how Frege makes the point, just with reference to the measure of a rectilinear distance by a Cauchy-series (Frege, 1903, §II.76; Frege, 2013, p. 881):

First we must know the magnitude-ratios, the real numbers; then we can discover how to determine ratios by means of fundamental series [i.e., Cauchy series][...]. Enlisting geometry is crucial since it is in this way that one appropriates the content at which all endeavors are directed. The crux is then to be found in geometry, however, and Cantor's theory is in no way purely arithmetical.

53 Cf. footnote (52), above. 
And here is how it is resumed by Dummett (Dummett, 1991, p. 276):

Cantor has not only assumed the principal notion to be explained, but it has assumed it without sufficient generality. What is required is an explanation, not of a specific notion of a ratio of distances, but of the general notion of a ratio of quantities [or magnitudes, in our terminology] of some one type: real numbers can be presented as precisely such a ratio, without importing anything into the definition from outside arithmetic. ${ }^{54}$ Because Cantor's construction of the real numbers does not present them as ratios of quantities [better: magnitudes], he can no more than illustrate their use to give the magnitude of a quantity [better: the measure of a magnitude] case by case; and this has the consequence that he has to appeal to nonarithmetical notions (in his example to geometrical ones).

Taking real numbers to be pairs like $\langle n, \Lambda\rangle$ would have, possibly, served the case of the inclusion of real analysis within logic, but, just as it happens with Cantor's definition, it would have appeared too quick to Frege. However ingenious it might be, such a definition could not throw any light on the fundamental concern of explaining in general the intimate relation between real analysis and theories of magnitudes; nor would it help accounting for centuries of mathematics spent trying to understand how numbers and magnitudes are reciprocally related.

This seems, indeed, the very aim of Frege: not including as much mathematics as possible within logic, but reconstructing mathematics, in connection to logic, so as to do justice to its millenarian vocation: that of providing us with a rigorous clarification of some basic scientific notions and of their mutual relations.

\$6. Concluding remarks. The attempt at giving a correct interpretation of Frege's foundational program has a long and very entangled history, which cannot be even cursorily surveyed here. It would, then, be illusory to try to conclude our article by situating our suggestion in such a large and intricate discussion. Just a few remarks are in order.

A useful way of recapitulating this debate is offered by Jeshion (2001). Jeshion reconstructs from the literature and then surveys three rationales that may have motivated this program (ibidem, pp. 939-940). Two of them, respectively, ascribed to Kitcher (1979) and Weiner $(1984,1990)$, make it an eminently philosophical program: one takes its purpose to be that of "demonstrating the epistemological superiority of arithmetical knowledge", under the assumption that "the logical source alone is capable of producing knowledge possessing self-evidence, certainty, and clarity"; the other takes its purpose to be that of "determining the epistemological sources of our arithmetical knowledge", thereby establishing whether they are $a$ priori or a posteriori, analytic or synthetic. The third, ascribed to Benacerraf (1981), makes it an eminently mathematical program, aiming at proving "whatever admits of proof", under the assumption that if a truth admits of proof it "ought to be proved".

Jeshion argues that these rationales are all either partial or incorrect, mainly because they make no case of Frege's twofold conception of self-evident, both as "selbstverstandlich", namely "foundationally secure" (neither "grounded on other truths" nor "in need of proof"), and as "einleuchten", namely such that "grasping their content" is sufficient for

54 Here Dummett seems to take arithmetic to be a general theory of numbers, including real ones, and not just the theory of natural numbers. 
"rational recognition of their truth" (Jeshion, 2001, p. 939). Integrating this concern leads, according to Jeshion, to conclude that Frege's program is motivated by a more articulated rationale, that she labels 'Euclidean', namely by the aim of making "primitive truth of mathematics" both selbstverstandlich and einleuchten, and the "relations of epistemic justification in a science" apt to mirror "the natural ordering of truths" (Jeshion, 2001, pp. 944 and 961). Jeshion takes this rationale to be both mathematical and epistemological: she takes Benacerraf to be correct "in holding that Frege's interests in establishing logicism were in many ways on a par with his mathematical contemporaries' interest in foundational projects", but also submits that "there is no denying that Frege had serious independent epistemological motivations" (ibidem, p. 944).

It would surely be dangerous to deny the latter, and it is far from our purpose to contradict Jeshion's analysis of Frege's notion of self-evidence (cf. Shapiro (2009) for a discussion). Let us notice, however, that in the light of Frege's actual formal achievements - the definition of natural numbers and of complete domains of magnitudes, and the proofs of the fundamental theorems of arithmetic - the only relevant testing bench for the claim that Frege was guided from Jeshion's Euclidean rationale concerns Basic Law V (unless one were willing to ascribe to Frege the though that explicit definitions are primitive truth: a view that, in any case, could not be incorporated in this rationale without much further work), which is, indeed, quite problematic for this view. ${ }^{55}$

What we want to focus on, however, is not so much Basic Law V, but rather the way Frege should have defined real numbers, in agreement with the idea that they are ratios of magnitudes. As we have already observed above (p. 135), even supposing that he could have appropriately proved that a domain of magnitudes necessarily exists, no explicit definition could have brought him from his definition of such domains to the introduction of ratios on them: this would have required an abstraction principle, that is, a new law having the same logical form of Basic Law V, presumably rephrasing Euclid's definition of proportionality.

Would Frege have considered this new law both selbstverstandlich and einleuchten? This would have been quite strange, in light of the more than bi-millenary discussion about the suspected inappropriateness of this definition as a ground for the theory of proportions.

Would he rather have bet for future clarifications or future selbstverstandlich and einleuchten ways to define real numbers as ratios of magnitudes? ${ }^{56}$ It may be quite pretentious to deny this (there are, after all, more things in heaven and earth than dreams in any philosophy). But even if this was the case, it would be just enough to suggest that what moved Frege was more to be able to provide an appropriate architecture of mathematics connecting real analysis to a general theory of magnitudes, or theory of domains of

55 To be honest, Jeshion acknowledges this last point and tries to accommodate for it in a way which, we must confess, we find not wholly convincing. In particular, she takes on herself to explain how Frege could have admitted, under the pressure of Russell's paradox, to have "never concealed from [...] [himself] that [...] [Basic Law V] is not as einleuchtend as must properly be required of a logical law" (Frege, 1903, Nachwort, p. 253; Frege, 2013, p. 2532; Jeshion, 2001, p. 970), and allegedly solves the problem by suggesting both that "Frege could have held that the coincidence in sense of $\varepsilon . F \varepsilon=\varepsilon \cdot G \varepsilon$ and $\forall x(F x \Leftrightarrow G x)$ would, in time, come to seem as obvious as the other axioms" (Jeshion, 2001, pp. 970-971), and that he "could have regarded his Grundgesetze system as a first, yet necessary, step toward the development of a genuine logical foundation" (ibidem, p. 971).

$56 \mathrm{Cf}$. footnote (55), above. 
magnitudes, than to wait for the happy time in which an epistemically appropriate alternative (with respect to his general philosophical criteria) would have appeared.

This seems to us to reinforce the idea that Frege's motivation for suggesting to define real numbers as ratios of magnitudes was, primarily, a mathematical one. Does this bring grist to Benacerraf's mill? Possibly. But only if this mill is more meticulously built. For it seems to us a little too weak to argue that Frege merely wanted to prove what is both probable and in need of proof. Surely, he wanted this. But how? And is this all that he wanted? After all, he famously remarked, just at the beginning of Grundlagen, that "after we have convinced ourselves that a boulder is immovable, by trying unsuccessfully to move it, there remains the further question, what is it that supports it so securely?" (Frege, 1903, §2; Frege, 1950, p. 2). His (mathematical) problem, we suggest, was not only (and not primarily) that of proving truth, but also (and mainly) that of organizing and structuring theories.

The important question was how this should and could be done. Philosophy alone seems unable to provide the answer. It surely can (and has to) give general criteria for the choice. But, then, specific insights are necessary for implementing those criteria in particular cases. By way of example, consider how Dedekind describes, in his letter to Lipschitz of July 22th, 1876, the aim of his essay on real numbers (Dedekind, 1888; ?, vol. III, p. 476):

The whole tendency of my essay [on irrational numbers] [...] is to use cuts $[\ldots]$ to prove that on the sole basis of the foundation of the arithmetic of rational numbers, and also with no appeal to the rather obscure and complicated concept of magnitudes, irrational numbers can be defined at once and, what is most important, in their ness (continuity).

For him, the notion of a magnitude was so "obscure and complicated" to convince him to undertake an important foundational effort (possibly resulting in the most influential foundational achievement of modern mathematics) in order to avoid appealing to it. For Frege, elucidating it—by avoiding to define continuity (which essentially characterizes magnitudes) by means of some artificial construction based on rational numbers-was the crucial task that an appropriate foundation of real analysis required.

Is there any philosophical (epistemic, metaphysical, ontological) criterion for deciding who was right, if any? Founding mathematics, in its deepest essence, means to make and (mathematically) motivate choices like this. What we have tried to show here, is that this was a (mathematical) game that Frege definitively played.

§7. Acknowledgments. The authors wish to thank the audiences of seminars and conferences where previous drafts of the article were presented, and in particular Francesca Boccuni, Sébastien Gandon, Mario Gómez Torrente, and Jamie Tappenden, as well as the editors and referees of the Review of Symbolic Logic. Several issues in this article were discussed with our friend and colleague Bob Hale, and were often elicited by his illuminating works on this subject—although he would have probably disagreed with our conclusions. This is why we dedicate this essay to his memory.

\section{BIBLIOGRAPHY}

Batitsky, V. (2002). Some measurement-theoretic concerns about Hale's 'Reals by Abstraction'. Philosophia Mathematica, 10(3), 286-303.

Benacerraf, P. (1965). What numbers could not be. Philosophical Review, 74(1), 47-73.

Benacerraf, P. (1981). Frege: The last logicist. Midwest Studies in Philosophy, 6(1), 17-36. 
Boccuni, F. \& Panza, M. (Manuscript). On the logicality of Frege's definition of real numbers.

Currie, G. (1986). Continuity and change in Frege's philosophy of mathematics. In Haaparanta, L. and Hintikka, J., editors. Frege Synthetisized. Esays on the Philosophical and Foundational Work of Gottlob Frege. Dordrecht, Boston, Lancaster, Tokyo: D. Reidel, pp. 345-373.

Dedekind, R. (1888). Was sind und was sollen die Zahlen. Braunschweig: F. Vieweg und Sohn. Also in Dedekind, R. (1930-1932). Gesammelte Mathematische Werke (E. N. von Herausgegeben, R. Fricke, and Ö. Ore, editors), Vol. 3. Braunschweig: Vieweg, Chapter LI, 335-391.

Díez, J. A. (1997). A hundred years of numbers. An historical introduction to measurement theory 1887-1990. Studies in History and Philophy of Sciences, 28, 167-185, 237-265.

Dummett, M. (1991). Frege. Philosophy of Mathematics. Cambridge (Massachusetts): Harvard University Press.

Frege, G. (1884). Die Grundlagen der Arithmetik. Eine Logische Mathematische Untersuchung über den Begriff der Zahl. Breslau: Verrlag von W. Koebner.

Frege, G. (1893-1903). Die Grundgesetze der Arithmetick, Vol. I-II. Jena: Hermann Pohle.

Frege, G. (1903). Über die Grundlagen der Geometrie. Jahresbericht der Deutschen Mathematiker-Vereinigung, 12, 319-324, 368-375.

Frege, G. (1950). The Foundations of Arithmetic. A logico-mathematical enquiry into the concept of number (Translated by J. L. Austin, second revised edition). New York: Harper \& Brothers.

Frege, G. (1971). On the Foundations of Geometry and Formal Theories of Arithmetic. Edited and translated by Kluge, E.-H. W. New Haven: Yale University Press.

Frege, G. (1976). Nachgelassene Schriften und Wissenschaftlicher Briefwechsel (H. Hermes, H. Kambartel, and F. Kaulbach, editors). Hamburg: Felix Meiner Verlag.

Frege, G. (2013). Basic Laws of Arithmetic. Tranlated and edited by P. A. Ebert and M. Rossberg with C. Wright. Oxford: Oxford University Press.

Gandon, S. (2012). Russell's Unknown Logicism. Palgrave MacMillan.

Gauss, C. F. (1831). Announcement of the Commentatio secunda to the Theoria residuorum biquadraticorum. Gottingische gelehrte Anzeigen, 1, 625-638. Also in Gauss, C. F. (1863-1917). Werke, Vol. 2. Herausgegeben von der Königlichen Gesellchaft der Wissenschaften zu Göttingen. Göttingen: Dietrich, pp. 169-178.

Goldfarb, W. (2001). Frege's conception of logic. In Floyd, J. and Shieh, S., editors. Future Pasts: The Analytic Tradition in Twentieth-Century Philosophy, Oxford, New York: Oxford University Press, pp. 25-41.

Hale, B. (2000). Reals by abstraction. Philosophia Mathematica (III), 8, 100-123.

Hale, B. (2002). Real numbers, quantities, and measurement. Philosophia Mathematica, 10(3), 304-323.

Hale, B. (2016). Definitions of numbers and their applications. In Ebert, P. A. and Rossberg, M., editors. Abstractionism: Essays in Philosophy of Mathematics, Oxford, New York: Oxford University Press, pp. 332-349.

Hankel, H. (1687). Theorie der Complexen Zahlensysteme [...]. Theil I der Vorlesugen über die Complexen Zahlen [... ] in Zwei Theilen. Leipzig: Leopold Voss.

Helmholtz, H. (1868). Ueber die Thatsachen, die der Geometrie zum Grunde liegen. Nachrichten von der Königlichen Gesellschaft der Wissenschaften [...], 9, Juni 3, pp. 193-221.

Helmoltz, H. v. (1887). Zählen und Messen, erkenntnisstheoretisch betrachtet. In Philosophische Aufsätze, Eduard Zeller zu seinem fünfzigjährigen Doctorjubiläum gewidmet, Leipzig: Fues' Verlag, pp. 17-52. 
Hölder, O. (1901). Die axiome der quantität und die lehre vom mass. Berichte über die Verhandlungen der Königlich Sächsischen Gesellschaft der Wissenschaften zu Leipzig, mathematisch-physischen Classe, 53, 1-64.

Jeshion, R. (2001). Frege's notions of self-evidence. Mind, 110(440), 937-976.

Kant, I. (1998). Critique of Pure Reason. Cambridge: Cambridge University Press. Tranlated and edited by P. Guyer and A. W. Wood.

Kitcher, P. (1979). Frege's epistemology. Philosophical Review, 88(2), 235-262.

Krantz, D. H., Luce, R. D., Suppes, P., \& Tversky, A. (1971-1990). Foundations of Measurement, Vol. 3. New York: Acedemic Press.

Kutschera, F. v. (1966). Freges begründung der analysis. Archiv für mathematische Logik und Grundlagenforschung, 9, 102-111.

MacFarlane, J. (2002). Frege, Kant, and the logic in logicism. The Philosophical Review, 111(1), 25-65.

McCallion, P. (2016). On Frege's applications constraint. In Ebert, P. A. and Rossberg, M., editors. Abstractionism: Essays in Philosophy of Mathematics. Oxford, New York: Oxford University Press, pp. 311-322.

Menge, H. (editor) (1846). Euclidis Data. Lipsiæ: B. G. Tuebneri.

Mill, J. S. (1872). A System Of Logic, Ratiocinative And Inductive [...], Eight Edition, Vol. 2. London: Longmans, Green, Reader, and Dyer.

Mill, J. S. (1877). System der Deductiven und Inductiven Logik [...]. In's Deutsche überetragen von J. Schiel. Vierte deutsche nach der Achten des Originals erweiterte Auflage, Vol. 2. Braunschweig: Friedrich Vieweg und Sohn.

Panza, M. (2016). Abstraction and epistemic economy. In Costreie, S., editor. Early Analytic Philosophy. New Perspective on the Tradition, Springer, Cham, Heidelberg, pp. 387-428.

Panza, M. (2018). Was Frege a logicist for arithmetic. In Coliva, A., Leonardi, P., and Moruzzi, S., editors. Eva Picardi on Language, Analysis and History. London, New York: Palgrave Macmillan, pp. 87-112.

Peacocke, C. (2015). Magnitudes: Metaphysics, explanation, and perception. In MoyalSharrock, D., Munz, V., and Coliva, A., editors. Mind, Language and Action: Proceedings of the $36^{\text {th }}$ International Wittgenstein Symposium, Berlin, Munich, Boston: De Gruyter, pp. 357-387.

Riemann, B (1866-1867). Ueber die Hypothesen, welche der Geometrie zu Grunde liegen. Abhandlungen der Königlichen Gesellshaft der Wissenshaften zu Göttingen, 13, 133150.

Riemann, B. (2016). Bernhard Riemann. On the Hypotheses Which Lie at the Bases of Geometry. Switzerland: Birkhäuser, Springer International Publishing. Edited by Jürgen Jost.

Russell, B. (1903). The Principles of Mathematics. Cambridge: Cambridge University Press.

Russell, B. \& Whitehead, A. N. (1910-1913). Principia Mathematica, Vol. 3. Cambridge: Cambridge University Press.

Sereni, A. (manuscript). On the Philosophical Significance of Frege's Constraint.

Shapiro, S. (1997). Philosophy of Mathematics: Structure and Ontology, Vol. 2. Oxford: Oxford University Press.

Shapiro, S. (2000). Frege meets dedekind: A Neologicist treatment of real analysis. Notre Dame Journal of Formal Logic, 4, 317-421.

Shapiro, S. (2009). We hold these truths to be self-evident: But what do we mean by that? Review of Symbolic Logic, 2(1), 175-207. 
Simons, P. (1987). Frege's theory of real numbers. History and Philosophy of Logic, 8 , 25-44. Also in Demopoulos, W. (1995). Frege's Philosopjy of Mathematics. Cambridge, MA: Harvard University Press, pp. 358-383.

Snyder, E., Samuels, R., \& Shapiro, S. (2018). Neologicism, Frege's constraint, and the Frege-Heck condition. Noûs, doi: 10.1111/nous.12249.

Steiner, M. (1998). The Applicability of Mathematics as a Philosophical Problem. Cambridge (Massachusetts): Harvard University Press.

Steiner, M. (2005). Mathematics: Application and applicability. In Shapiro, S., editor. Oxford Handbook of the Philosophy of Mathematics and Logic, Oxford, New York: Oxford University Press, pp. 625-650.

Taisbak, C. M. (2003). $\Delta \mathrm{E} \Delta$ OMENA. Euclid's Data or The Importance of Being Given. Copenhagen: Museaum Tusculanum Press.

Weiner, J. (1984). The philosopher behind the last lsogicist. Philosophical Quarterly, 34(136), 242-264.

Weiner, J. (1990). Frege in Perspective. Cornell University Press.

Wright, C. (2000). Neo-Fregean foundations for real analysis: Some reflections on Frege's constraint. Notre Dame Journal of Formal Logic, 41(4), 317-334.

CNRS, IHPST

13 RUE DU FOUR

PARIS 75006, FRANCE (CNRS AND UNIV. PARIS 1, PANTHÉON-SORBONNE),

E-mail:marco.panza@univ-paris1.fr

and

CHAPMAN UNIVERSITY

ONE UNIVERSITY DRIVE

ORANGE, CA 92866, USA

E-mail: panza@chapman.edu

\section{SCUOLA UNIVERSITARIA SUPERIORE IUSS PAVIA \\ NETS CENTER \\ PIAZZA DELLA VITTORIA, 15 \\ 27100 PAVIA, ITALY}

E-mail: andrea.sereni@iusspavia.it 